Original article

\title{
Interplay between parasite cysteine proteases and the host kinin system modulates microvascular leakage and macrophage infection by promastigotes of the Leishmania donovani complex
}

\author{
Erik Svensjö ${ }^{\text {a }}$, Paulo R. Batista ${ }^{a}$, Claudia I. Brodskyn ${ }^{\mathrm{b}}$, Robson Silva ${ }^{\mathrm{b}}$, Ana Paula C.A. Lima ${ }^{\mathrm{a}}$, \\ Verônica Schmitz ${ }^{\text {a }}$, Elvira Saraiva ${ }^{\text {c }}$, João B. Pesquero ${ }^{\text {d }}$, Marcelo A.S. Mori ${ }^{\mathrm{d}}$, \\ Werner Müller-Esterl ${ }^{\mathrm{e}}$, Julio Scharfstein ${ }^{\mathrm{a}, *}$ \\ ${ }^{a}$ Instituto de Biofísica Carlos Chagas Filho, Universidade Federal do Rio de Janeiro, C.C.S., Ilha do Fundão, Rio de Janeiro, 21949-900 RJ, Brazil \\ ${ }^{\mathrm{b}}$ Centro de Pesquisa Gonçalo Moniz, LIP, R. Valdemar Falcão, 121. Salvador, Bahia 40295-001, Brazil \\ c Instituto de Microbiologia Paulo de Góes, Universidade Federal do Rio de Janeiro, C.C.S., Ilha do Fundão, Rio de Janeiro, 21949-900 RJ, Brazil \\ ${ }^{\mathrm{d}}$ Departamento de Biofísica, Universidade Federal do Estado de São Paulo (UNIFESP) Escola Paulista de Medicina, São Paulo, Brazil \\ e Institute for Biochemistry II, University of Frankfurt Medical School, Frankfurt, Germany
}

Received 11 February 2005; accepted 16 June 2005

Available online 08 September 2005

\begin{abstract}
Kinins, the vasoactive peptides proteolytically liberated from kininogens, were recently recognized as signals alerting the innate immune system. Here we demonstrate that Leishmania donovani and Leishmania chagasi, two etiological agents of visceral leishmaniasis (VL), activate the kinin system. Intravital microscopy in the hamster cheek pouch showed that topically applied promastigotes induced macromolecular leakage (FITC-dextran) through postcapillary venules. Peaking at $15 \mathrm{~min}$, the parasite-induced leakage was drastically enhanced by captopril (Cap), an inhibitor of angiotensin-converting enzyme (ACE), a kinin-degrading metallopeptidase. The enhanced microvascular responses were cancelled by HOE-140, an antagonist of the $\mathrm{B}_{2}$ bradykinin receptor $\left(\mathrm{B}_{2} \mathrm{R}\right)$, or by pre-treatment of promastigotes with the irreversible cysteine proteinase inhibitor $N$-methylpiperazine-urea-Phe-homoPhe-vinylsulfone-benzene (N-Pip-hF-VSPh). In agreement with the above-mentioned data, the promastigotes vigorously induced edema in the paw of Cap-treated J129 mice, but not Cap- $\mathrm{B}_{2} \mathrm{R}^{-} /{ }^{-}$mice. Analysis of parasite-induced breakdown of high molecular weight kininogens (HK), combined with active site-affinity-labeling with biotin$\mathrm{N}-\mathrm{Pip}-\mathrm{hF}-\mathrm{VSPh}$, identified 35-40 kDa proteins as kinin-releasing cysteine peptidases. We then checked if macrophage infectivity was influenced by interplay between these kinin-releasing parasite proteases, kininogens, and kinin-degrading peptidases (i.e. ACE). Our studies revealed that full-fledged $\mathrm{B}_{2} \mathrm{R}$ engagement resulted in vigorous increase of $L$. chagasi uptake by resident macrophages. Evidence that inflammatory macrophages treated with HOE-140 became highly susceptible to amastigote outgrowth, assessed $72 \mathrm{~h}$ after initial macrophage interaction, further suggests that the kinin/ $\mathrm{B}_{2} \mathrm{R}$ activation pathway may critically modulate inflammation and innate immunity in visceral leishmaniasis.
\end{abstract}

(C) 2005 Elsevier SAS. All rights reserved.

Abbreviations: ACE, angiotensin-converting enzyme; BK, bradykinin; BSA, bovine serum albumin; Cap, captopril; CBZ-Phe-Arg-MCA, carbobenzoxyphenylalanyl-arginyl-7-amido-4-methylcoumarin; CP, cysteine proteinases; DTT, ditiothreitol; E-64, L-trans-epoxysuccinyl-leucylamido-(4-guanidino)butane; HCP, hamster cheek pouch; HK, high molecular weight kininogen; N-Pip-F-hF-VSPh or K11777, N-methylpiperazine-Phe-homoPhe-vinylsulfone-benzene; pHMG, promastigotes homogenates; TK, tissue kallikrein; VL, visceral leishmaniasis1. Introduction.

* Corresponding author. Tel.: +55212209 6591; fax: +552122808193.

E-mail address: scharf@biof.ufrj.br (J. Scharfstein). 


\section{Introduction}

Transmitted by sandflies, the parasitic protozoa of the genus Leishmania are the etiological agents of cutaneous, mucocutaneous or visceral diseases in humans. Differences in Leishmaniasis species and host genetic composition underlie the diverse clinical manifestations of Leishmaniasis.

The life cycle of all Leishmania species involves two morphological forms - the amastigotes and the promastigotes. Mammalian infection is initiated when the sand-flies regurgitate following blood feeding, releasing metacyclic promastigotes in superficial layers of the dermis. At this time, the mechanical action of the sand fly proboscis provokes lacerations of blood capillaries in the host. Hemorrhages are intensified by the combined action of multiple anti-hemostatic factors and vasodilating substances present in the sand fly saliva [1]. Within minutes, the promastigotes are phagocytosed by macrophages, the internalization process being facilitated by complement receptor-mediated recognition of surfacebound $\mathrm{C} 3 \mathrm{~b}$ and/or C3bi opsonins [1-4]. After reaching the phagolysosomes, the promastigotes transform into amastigotes, the parasite replicating stages. Upon macrophage cell death, large numbers of infective amastigotes are released to the extracellular spaces. Recent studies in humans and mice suggest that innate immunity and pathological outcome in Leishmania infection is influenced by host responses to the sand fly vector. For instance, salivary products of Phlebotomus papatasi saliva products may induce IL-4 and IL-5, i.e. cytokines that down-modulate immune responses to Leishmania major [5]. Another study in patients infected with Leishmania chagasi showed that individuals that undergo seroconversion to sand fly saliva antigens are more prone to develop efficient cellular immune responses (delayed type hypersensitivity; DTH) against the parasite [6]. The complexity of the host-vector-parasite interplay is highlighted by the finding that increased blood flow in individuals that mount DTH responses to sand fly saliva antigens improves blood feeding by $P$. papatasi [7].

Visceral leishmaniasis (VL), also known as Kalazar, is caused by Leishmania (Leishmania) donovani in the Old World, by $L$. (L.) infantum in the Southeast of Europe and Mediterranean area and by $L$. $(L$. $)$ chagasi in the New World. Dogs are a common animal reservoir of the Leishmania species that cause visceral leishmaniasis. The incubation period of visceral leishmaniasis ranges from 2 to 4 months. The disease may present an acute, subacute or chronic evolution, but most infected individuals remain completely asymptomatic (reviewed in [8]). The classical manifestations of VL are fever, cough, weight loss, weakness, diarrhea or dysentery and abdominal swelling. Patients also present anemia, edema, bleeding episodes, impaired cellular immunity and severe hepatosplenomegaly [8]. The determinants of visceralization in infected humans are not well understood. Studies in mice revealed that visceralization starts with a self-limiting infection in the liver, usually controlled at expense of moderate TNF- $\alpha$ production [9]. After involving the spleen, the infec- tion in mice becomes persistent, causing a life-long splenomegaly associated with exacerbated TNF- $\alpha$ production [9]. Immunohistochemical studies performed with high dose of intravenously injected amastigotes showed that the parasites are initially cleared from the circulation by the heterogenous macrophage sub-populations present in the spleen marginal zone [10]. Low dose infection models involving intradermal inoculation of $L$. donovani promastigotes into susceptible mice [11] indicated that parasite clearance from skin and liver may depend on infiltration and activation of $\mathrm{CD}^{+}$and $\mathrm{CD}^{+}$ $\mathrm{T}$ cells while the persisting infection in the spleen and draining lymph node correlates with enhanced production of Th1/Th2 cytokines (interleukin-4 [IL-4], IL-10, and gamma interferon) [11].

Here we studied the involvement of the kinin system in inflammatory responses evoked by L. donovani and L. chagasi. Structurally related to the nonapeptide bradykinin (BK), the term "kinins" is a general designation for the group of potent vasoactive peptides liberated from an internal segment of their plasma protein precursors, high and/or lowmolecular weight kininogens (HK/LK) [12]. Although classically associated with acute inflammatory responses (e.g. increased blood flow, edema formation, vasodilatation and pain sensations), BK or for that matter, lysyl-BK, were recently identified as potent stimulators of dendritic cell maturation, an effect coupled to IL-12-driven polarization of Th1type responses [13]. Tissue injury caused by physical trauma or noxious substances can lead to BK or Lysyl-BK excision from $\mathrm{HK} / \mathrm{LK}$ by the respective action of the serine proteases, plasma and tissue kallikrein [12]. In inflammatory conditions, oxidized forms of kininogens may be cleaved by the concerted action of neutrophil elastase and mast cell tryptase, liberating Met-Lys-bradykinin [14]. Once liberated, kinins act in a paracrine mode, activating a broad range of host cell types (e.g. endothelial cells, epithelial cells, neurons and dendritic cells) through the constitutively expressed $\mathrm{B}_{2}$ bradykinin receptor $[13,15]$. Alternatively, they undergo further processing by carboxypeptidases $\mathrm{M} / \mathrm{N}$, generating [des-Arg]-kinins, which are agonists for the $\mathrm{B}_{1}$ kinin receptor, upregulated during inflammation [16]. The duration of the effects of kinins on their G-protein coupled receptors is normally limited by the degradative action of several metallopeptidases, particularly, angiotensin-converting enzyme (ACE, also known as kininase II), a di-peptidylpeptidase expressed at high levels on the vascular endothelium.

Activation of the kinin pathway has been implicated in the dissemination of several bacterial pathogens $[17,18]$. Inflammation induced by Porphyromonas gengivalis, the etiological agent of periodontitis, is partly driven by the kininreleasing activity of a cysteine protease, gingipain $[19,20]$. Another example of a pathogen that relies on a cysteine protease to liberate kinins from kininogens came from our own investigations [21-24] on the cellular activation pathways underlying inflammation and host cell invasion by Trypanosoma cruzi, the intracellular parasitic protozoan that causes Chagas' disease. Here we report results from studies in two 
animal models, hamster cheek pouch and mouse paw, demonstrating that L. donovani or L. chagasi promastigotes share with $T$. cruzi the ability to activate the kinin system. Further, we will present data linking regulation of the kinin system to pathways that modulate inflammation and macrophage susceptibility to infection by $L$. donovani and L. chagasi promastigotes.

\section{Materials and methods}

\subsection{Parasites}

L. chagasi promastigotes (MHOM/BR00/MER/Strain2) were cultivated in Schineider's insect Medium (Sigma Chemical Co., St Louis, MO, USA) supplemented with $20 \%$ inactivated FCS, L-glutamine ( $2 \mathrm{mM})$, penicillin $(100 \mathrm{U} / \mathrm{ml})$, streptomycin $(100 \mu \mathrm{g} / \mathrm{ml})$ at $23{ }^{\circ} \mathrm{C}$ for $5-7$ days when the parasites reached the stationary-phase. The parasites used in macrophage infection assays were washed $3 \times$ times with saline at $2500 \mathrm{rpm}$ for $10 \mathrm{~min}$, resuspended in RPMI medium and adjusted to $5 \times 10^{7}-10^{8}$ per ml. L. donovani Sudan (LD 1S/MHOM/SD/00-strain 1S) was cultured in BHI medium containing 10\% FCS (GIBCO BRL, Tulsa, OK). L. chagasi metacyclics were prepared from 5 days stationary-phase cultures using a density-gradient method [25]. Briefly, promastigotes were washed and resuspended in Hanks, then layered on a two-step Ficoll gradient ( $2 \mathrm{ml}$ of $5 \%$ and $4 \mathrm{ml}$ of $10 \%$ ) and centrifuged at $1300 \times g$ for $10 \mathrm{~min}$ at room temperature. Based on morphological criteria, the L. chagasi promastigotes recovered from the second $10 \%$ step had $70-90 \%$ of metacyclics. $40 \%$ of this $L$. chagasi population corresponds to the subset of metacyclics that is resistant to complementmediated lysis (25\% fresh human serum) while log-phase $L$. chagasi promastigotes are $100 \%$ lyzed under these conditions. Assays with L. donovani promastigotes were performed with promastigotes isolated from 7-day-old stationaryphase cultures, after adjusting the cell suspension to $5 \times 10^{7}-$ $10^{8}$ per $\mathrm{ml}$ in phosphate buffered saline. Cellular homogenates obtained from L. donovani promastigotes (pHMG) were obtained by subjecting the parasite suspension to four to five freezing-thawing cycles followed by centrifugation at $1300 \times g$ for $10 \mathrm{~min}$ in PBS before being used in the studies described below.

\subsection{Intravital microscopy of the hamster cheek pouch}

Hamsters were anesthetized by intraperitoneal injection of sodium pentobarbital supplemented with i.v. $\alpha$-chloralose $(2.5 \% \mathrm{~W} / \mathrm{V}$, solution in saline) through a femoral vein catheter. A tracheal cannula (PE 190) was inserted to facilitate spontaneous breathing and the body temperature was maintained at $37^{\circ} \mathrm{C}$ by a heating pad monitored with a rectal thermistor. The hamster cheek pouch (HCP) was prepared and used for intravital microscopy as previously reported [26,27]. Briefly, the cheek pouch was everted and mounted on a micro- scope stage and an area of about $1 \mathrm{~cm}^{2}$ was prepared for intravital microscopy observations of the microcirculation. Thirty min after the preparation was completed, fluorescein-labeled dextran (FITC-dextran, MW = $150 \mathrm{kDa} ; 250 \mathrm{mg} / \mathrm{Kg}$ body weight) was injected intravenously as a macromolecular tracer. The cheek pouch was continuously superfused with a HEPES-bicarbonate-buffered saline solution $\mathrm{pH} 7.4$ (composition in mM: $110.0 \mathrm{NaCl}, 4.7 \mathrm{KCl}, 2.0 \mathrm{CaCl}_{2}, 1.2 \mathrm{MgSO}_{4}$, 18.0 $\mathrm{NaHCO}_{3}, 15.39 \mathrm{HEPES}$ and $14.61 \mathrm{Na} \mathrm{HEPES}$ ) at a constant rate of $6 \mathrm{ml} / \mathrm{min}$. The superfusion solution was continuously bubbled with a $5 \% \mathrm{CO}_{2}$ and $95 \% \mathrm{~N}_{2}$-mixture to maintain a low and physiological level of oxygen around the cheek pouch. A heater device was adjusted to maintain a temperature of $35^{\circ} \mathrm{C}$ of the superfusion solution. The microvascular permeability increase for large molecules (plasma leakage) was quantified by counting sites with extravasation of fluorescent plasma (leaky sites $=$ leaks) at postcapillary venules at 2-5 min intervals after topical application of $L$. donovani promastigotes or L. chagasi metacyclic promastigotes. Experiments were started by applying $0.5 \mathrm{ml}$ of promastigotes $\left(50 \times 10^{6}\right)$ or equivalent doses of parasite homogenates (pHMG), to the HCP, for 10 min during interrupted superfusion. A second application was made $30 \mathrm{~min}$ later when the permeability increase observed after the first application had been resolved and the cheek pouch had been cleared of FITCdextran. The first series (I) of experiments consisted of four groups of 6-11 animals each and involved two applications of $L$. donovani promastigotes in each hamster applied at a 30 min interval. In group I.1 (control), the HCP tissues were superfused with normal medium. In group I.2, the superfusate was supplemented with $1 \mu \mathrm{M}$ of the ACE inhibitor captopril (Cap) (Sigma Chemical Co., St. Louis) for 5-10 min. Group I.3 consisted of superfusate containing Cap and $0.5 \mu \mathrm{M}$ (final concentrations) of HOE-140, a $\mathrm{B}_{2} \mathrm{R}$ antagonist (Aventis Pharmaceuticals, Bridgewater, NJ, USA), added $10 \mathrm{~min}$ prior to promastigote application. In group I.4, parasites were pre-treated with $10 \mu \mathrm{M}$ of N-Pip-F-hF-VSPh (referred to as K11777 in the Figures), an irreversible cysteine protease inhibitor originally developed against cruzain [28]; separate batches were kindly donated by Dr. J.H. McKerrow through Dr. J. Engel (UCSF, San Francisco, CA) and by Dr. J. Palmer (Axis Pharmaceutical). As controls for treatment with N-PipF-hF-VSPh, the promastigotes were pre-incubated with buffer containing the same final concentrations of DMSO, used as the solvent. Finally $30 \mathrm{~min}$ after the second parasite application $(t=60 \mathrm{~min})$, we checked if the HCP responsiveness was still maintained by adding $250 \mathrm{nM}$ BK and $1 \mu \mathrm{M}$ Cap (final concentrations) to the preparation for $5 \mathrm{~min}$. This final functional test with $\mathrm{BK}$ could not be done with $\mathrm{HCP}$ preparations that previously received HOE-140 because this high affinity antagonist cannot be efficiently removed from tissues by washings. Rather, at the end of these particular assays, the HCP preparation was challenged with $5 \mu \mathrm{M}$ histamine for 5 min. Experiments using L. chagasi metacyclic promastigotes (series II.1-3), were performed as described above for $L$. donovani promastigotes, except for that the second applica- 
tion of L. chagasi parasites was not made. Series III consisted of four groups of experiments of five to six animals each, involving two consecutive topical applications of $\mathrm{pHMG}$ (equivalent to $50 \times 10^{6}$ promastigotes) with 30 min intervals. In the group III.1 (control), the HCP was superfused with normal medium. Group III. 2 consisted of superfusate supplemented with $1 \mu \mathrm{M}$ Cap while in group III.3, Cap was combined with $0.5 \mu \mathrm{M}$ HOE-140 (III.3). All the experiments were terminated 30 min after the second homogenate application ( $t=60 \mathrm{~min})$, by applying BK $(250 \mathrm{nM})$ and Cap $(1 \mu \mathrm{M})$ for $5 \mathrm{~min}$. Hamsters were randomly allocated to the different treatment schedules within each experimental series. Data are presented as mean \pm S.E.M. Statistical evaluation (between groups) was performed with ANOVA followed by a Student's $t$-test. A $P$-value of 0.05 or less was considered to indicate a statistically significant difference.

\subsection{Mouse paw edema}

Experiments were conducted as previously described [24], using either male Balb/c, male wild-type (WT) J129, or male $\mathrm{J} 129 \mathrm{~B}_{2} \mathrm{R}^{-/}$mice [29], each weighing 35-50 g, housed at $22 \pm 2{ }^{\circ} \mathrm{C}$ with a 12-h light-dark cycle. Some of the animals were pre-treated i.p. with Cap (Sigma, St. Louis, MO), at $4 \mathrm{mg} / \mathrm{kg}, 1 \mathrm{~h}$ before the injection of $L$. donovani promastigotes. Animals under light ether anesthesia received an i.d. injection of $1 \times 10^{6}$ promastigotes suspended in $10 \mu \mathrm{l}$ of PBS. As a control, the contralateral paw received the same volume of PBS. The specificity of the $B_{2} R$ responses was examined by dorsal subcutaneous injection of HOE-140 $(200 \mu \mathrm{g} / \mathrm{kg})$ $1 \mathrm{~h}$ before parasite inoculation. pHMG treatment with N-PipF-hF-VSPh (K11777) was performed as described for living parasites in the previous section. Edema was measured with the aid of a plethysmometer at time intervals after i.d. injections and was expressed in microliter $(\mu \mathrm{l})$ (the difference in volume between the test and control paws). Statistical evaluations were determined by one-way ANOVA with a $P=0.05$ significance level. Experiments were made in accordance with current guidelines for the care of laboratory animals and ethical guidelines for experiments in conscious animals.

\subsection{Biochemical and enzymatic characterization of promastigote cysteine proteases}

After harvesting the L. donovani promastigotes at day 7 or L. chagasi metacyclics at day 5 , the cells were washed twice in Hank's balanced salt saline (BSS) and lyzed in $20 \mathrm{mM}$ $\mathrm{Na}_{2} \mathrm{HPO}_{4}, 150 \mathrm{mM} \mathrm{NaCl}, \mathrm{pH} 7.2$ (PBS) containing $1 \%$ $\mathrm{NP}-40$, on ice for $30 \mathrm{~min}$. The soluble fraction was collected by centriffugation at $13,000 \times g$ for $10 \mathrm{~min}$ and the protein concentration of the lysates was determined using the DcProtein kit (Bio-Rad). The secretion products were obtained upon incubation of washed cells $\left(10^{8}\right.$ per $\left.\mathrm{ml}\right)$ in BSS for $30 \mathrm{~min}$. at $26^{\circ} \mathrm{C}$. The supernatant was collected by centrifugation at $3000 \times g$ for $5 \mathrm{~min}$ and filtered in a $0.22 \mu \mathrm{m}$ acetate membrane. The peptidase activity present in lysates $(1 \mu \mathrm{g} / \mathrm{ml}$ protein) or in the supernatants (1:20 dilution) was monitored by the hydrolysis of $5 \mu \mathrm{M}$ CBZ-Phe-Arg-MCA in $50 \mathrm{mM}$ $\mathrm{Na}_{2} \mathrm{HPO}_{4}, 200 \mathrm{mM} \mathrm{NaCl}, 5 \mathrm{mM}$ EDTA, pH 6.5, 5 mM DTT, $0.5 \% \mathrm{DMSO}$, in a Hitachi-F4500 fluorimeter at $380 \mathrm{~nm}$ excitation, $440 \mathrm{~nm}$ emission. The initial velocities were calculated by linear regression of the substrate hydrolysis curves. Where indicated, E-64 was added at $30 \mu \mathrm{M}$ and N-Pip-F-hF$\mathrm{VSPh}(\mathrm{K} 11777)$ at $10 \mu \mathrm{M}$, final concentrations. Active-site labeling of the parasite cysteine proteinases was carried out by incubating the parasite lysates $(30 \mu \mathrm{g})$ in $50 \mathrm{mM} \mathrm{Na}$ acetate buffer, $200 \mathrm{mM} \mathrm{NaCl}, 5 \mathrm{mM}$ EDTA, pH 5.5, 5 mM DTT or, alternatively, as specificity controls, the same buffer supplemented with $30 \mu \mathrm{M}$ of E-64, for $10 \mathrm{~min}$. at room temperature. Labeling of the proteases was achieved by addition of $10 \mu \mathrm{M}$ of biotin-aminocaproyl-N-Pip-urea-F-hF-VSPh (biotin-N-Pip-urea-F-hF-VSPh) kindly donated by Dr. J. Palmer (Axys Pharmaceutical, SF, US). The samples were incubated for $1 \mathrm{~h}$ at RT and then boiled in SDS-2ME lysis buffer. Proteins were resolved by SDS-PAGE and then transferred to nitrocellulose. The blotted membrane was treated with PBS, $0.05 \%$ Tween-20, 9\% non-fat milk, followed by incubation with extravidin-alkaline phosphatase (Sigma). Visualization of the reactive products in the blots was done according to manufacturer's instructions.

\subsection{Parasite-mediated processing of purified $\mathrm{HK}$}

Stationary-phase promastigotes harvested at day 7 were washed twice in saline. The cells were resuspended in HAM pH 6.5 supplemented with $10 \mu \mathrm{g} / \mathrm{ml}$ of HK (Calbiochem) and incubated for $1 \mathrm{~h}$ at $26^{\circ} \mathrm{C}$ in the presence of DTT. Protease involvement was studied by adding $10 \mu \mathrm{M}$ of N-Pip-F-hFVSPh (K11777) or $50 \mathrm{mM}$ of EDTA prior to parasite addition. Toxicity was minimal, since the mobility of flagellates was the same as in controls, at the end of the incubation period. Positive controls for HK processing were performed with tissue kallikrein (TK) at $2 \mu \mathrm{g} / \mathrm{ml}$. The cell-free supernatants from these suspensions were boiled in SDS-lysis buffer, under reducing conditions. Western blotting was carried out using MBK3 (ascites, tested at 1:5000), an IgG1 mAb directed against the BK epitope of HK/LK [30]. Reaction products on blots were identified using anti-mouse IgG conjugated to alkaline phosphatase, as described above.

\subsection{Macrophage infection assays}

To determine the influence of kinin system on parasite uptake, monolayers of resident mouse peritoneal macrophages $(\mathrm{BALB} / \mathrm{c})$ or spleen adherent cells (isolated from $\mathrm{BALB} / \mathrm{c}$ mice or hamster as described below) were prepared by plating the cells on glass coverslips. The interaction (hostparasite interaction ratio of $1: 5$ ) proceeded for $4 \mathrm{~h}$ at $37^{\circ} \mathrm{C}$ in RPMI-10\% FCS, or in serum-free RPMI, supplemented, or not, with Cap $(25 \mu \mathrm{M})$ and/or HOE-140 (100 nM). At the end of this period, the monolayers were fixed with methanol and 
stained by hematoxylin-eosin. A replica plate was used to investigate the influence of kinin system activation (during the $4 \mathrm{~h}$ interaction period) on extent of intracellular parasite survival, $72 \mathrm{~h}$ later. To this end, the macrophage monolayers that had been exposed to the parasites were washed at the end of the interaction period and RPMI-FCS was added for additional $72 \mathrm{~h}$, before being fixed and stained. Mouse resident peritoneal macrophages $(\mathrm{BALB} / \mathrm{c})$ were obtained by injecting 5-10 $\mathrm{ml}$ of saline into the peritoneal cavity. The cell suspension was washed in saline $3 \times$ at $1500 \mathrm{rpm}$ for $10 \mathrm{~min}$ and then resuspended in serum-free RPMI. After plating the cells on glass coverslips at $1 \times 10^{6}$ per well in 24 well polystyrene plates (Costar, NY, USA), they were incubated for $4 \mathrm{~h}$ at $37^{\circ} \mathrm{C}$ in a $5 \% \mathrm{CO}_{2}$ atmosphere. The non-adherent cells were removed by successive washes with saline, while the adherent cell population was incubated for 2 days in RPMI containing $10 \%$ of fetal calf serum. To study the effect of exogenous BK or HK on mouse (BALB/c) spleen adherent cells, we added increasing concentrations of BK (5-300 nM) or HK (5-300 nM) to serum-free RPMI medium, prior to addition of parasites. Where indicated, Cap $(25 \mu \mathrm{M})$ and/or HOE$140(100 \mathrm{nM})$ were added to RPMI-10\% FCS or serum-free RPMI, immediately before addition of washed promastigotes. To verify if parasite $\mathrm{CP}$ contribute to macrophage infection, promastigotes were pre-treated with N-Pip-hF$\mathrm{VSPh} / \mathrm{K} 11777(10 \mu \mathrm{M})$ with $10 \mathrm{~min}$ at RT. After removing the excess of the CP inhibitor by centrifugation $(2500 \mathrm{rpm}$, $10 \mathrm{~min}$ ), the K11777-treated promastigotes were then added to the monolayer of peritoneal macrophages, in the presence of RPMI-10\% FCS). Adherent spleen cells, either derived from hamster or BALB/c mice, were obtained after homogenizing the spleens (4-6) in $5 \mathrm{ml}$ of serum- free RPMI. After re-suspending the cells in RPMI medium (GIBCO, Grand Island, NY, USA), they were plated at $4 \times 10^{6}$ per well at glass coverslips in 24 well polystyrene plates (Costar, NY, USA) and incubated for $4 \mathrm{~h}$ at $37{ }^{\circ} \mathrm{C}$ in a $5 \% \mathrm{CO}_{2}$ atmosphere. After removing non-adherent cells, the adherent cells were further incubated for 5-7 days in RPMI medium supplemented with inactivated 10\% FCS, HEPES $(10 \mathrm{mM})$, L-glutamine $(2 \mathrm{mM})$, penicillin $(100 \mathrm{U} / \mathrm{ml})$, streptomycin (100 $\mu \mathrm{g} / \mathrm{ml})$. By flow cytometry, $59 \%$ of the mouse adherent spleen cells were CD11 $\mathrm{b}^{+}$. Thioglycollate (TG)-elicited macrophages from BALB/c (males) were isolated after inoculating $3 \mathrm{ml}$ of $3 \% \mathrm{TG}$ (Sigma). Three days latter, the exudate peritoneal cells were collected by two washes $(5 \mathrm{ml}$ each) of chilled PBS. The cells were then plated $\left(5 \times 10^{4}\right.$ cells per well $)$ on glass coverslips. After $24 \mathrm{~h}$ incubation in RPMI-10\% FCS, the monolayer of adherent cells was washed $3 \times$ with RPMI and the wells were filled with RPMI-10\% FCS. The interaction between promastigotes and TG-macrophages ( $3 \mathrm{~h}$ ) was carried out in RPMI-10\% FCS supplemented, or not, with Cap and/or HOE-140, as described above. Intracellular parasite numbers (per 100 cells) and \% infected cells were determined at $72 \mathrm{~h}$ post-infection. In these particular experiments, we used Bouin and Giemsa, respectively, to fix and stain monolayers of TG-macrophages. Statistical comparisons were made with the Kruskal-Wallis test. A $P$-value of $<0.05$ was considered to indicate statistically significant differences between means.

\section{Results}

\subsection{Promastigotes activate the kinin pathway in vivo}

Since mice are relatively resistant to infection by Leishmania species that cause visceral leishmaniasis, we started our investigations in the hamster, a susceptible host species that exhibits manifestations that mimic several aspects of the visceral human disease [31]. To study mechanisms underlying Leishmania-induced inflammation at early stages of infection, we turned to the hamster cheek pouch preparation (HCP), because it is a convenient model to study the dynamics of plasma exudation or macromolecular leakage by intravital microscopy [32]. The initial experiments (refer to Series I in Section 2) were performed by topically applying stationaryphase L. donovani promastigotes to the HCP during interrupted superfusion. First, we studied the effects of parasite addition in HCP superfused with normal medium (GroupI.1). Moderate (and transient) macromolecular leakage responses were consistently observed $15 \mathrm{~min}$ after $L$. donovani application. (Fig. 1A). Addition of HOE-140 did not prevent induction of this mild venular leakage reaction nor delay its evolution (data not shown), suggesting that it was driven by kininindependent mechanisms. We then reasoned that kinins eventually generated under these conditions were swiftly degraded by tissue metallopeptidases, such as ACE/kininase II. To address this possibility, the ACE inhibitor captopril (Cap) [33] was added to the superfusate. Indeed, the experiments (Group I.2) showed that permeability responses induced by L. donovani were heightened in Cap-HCP (Fig. 1A). First, the maximal response in the Cap group $(86 \pm 19$ leaks per $\left.\mathrm{cm}^{2}\right)$ was significantly $(P<0.05)$ higher than that seen in untreated control $\left(51 \pm 3\right.$ leaks per $\left.\mathrm{cm}^{2}\right)$. Second, addition of HOE-140 to the Cap-superfusate (Group I.3) reduced macromolecular leakage evoked by $L$. donovani $(35 \pm 13$ leaks per $\mathrm{cm}^{2}$ ) (Fig. 1A), indicating that the observed enhancement depended on activation of the constitutive $\mathrm{B}_{2} \mathrm{R}$. Analysis of the time course of macromolecular leakage at postcapillary venules indicated that it started within 10 min of parasite challenge in Cap-HCP, that is, the activation response evolved faster than in HCP superfused with normal medium. As with controls, the permeability responses observed in Cap-HCP consistently peaked at $15 \mathrm{~min}$, being resolved at approximately $30 \mathrm{~min}$. Although not recorded or measured, increased rolling and adhesion of leukocytes were observed in postcapillary venules prior to plasma leakage. A second application of promastigotes was made 30 min later, when the HCP has been cleared from extravasated FITC-dextran. At this time, virtually no leaks were observed (see arrow) indicating that the first application of the pathogen induced a state of tachyphylaxis or preconditioning. Interestingly, a prominent leak- 
A

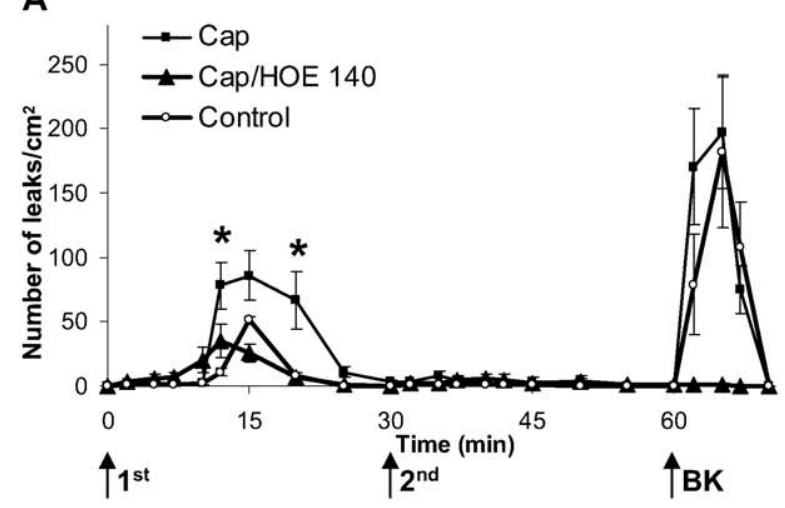

B

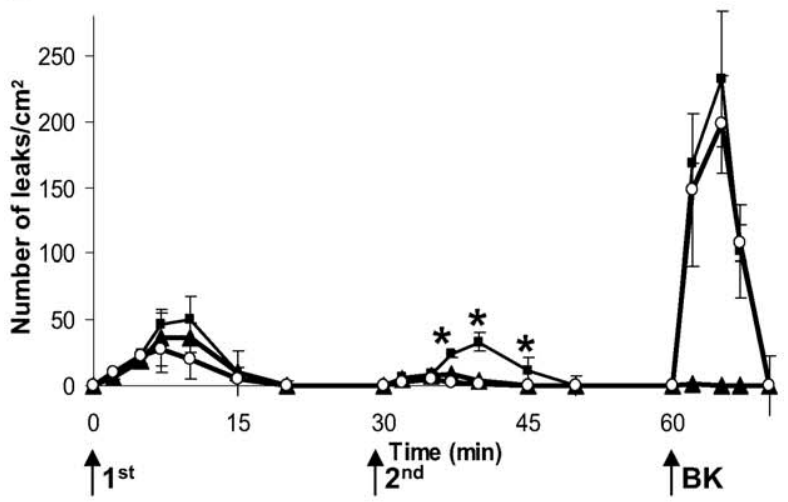

C

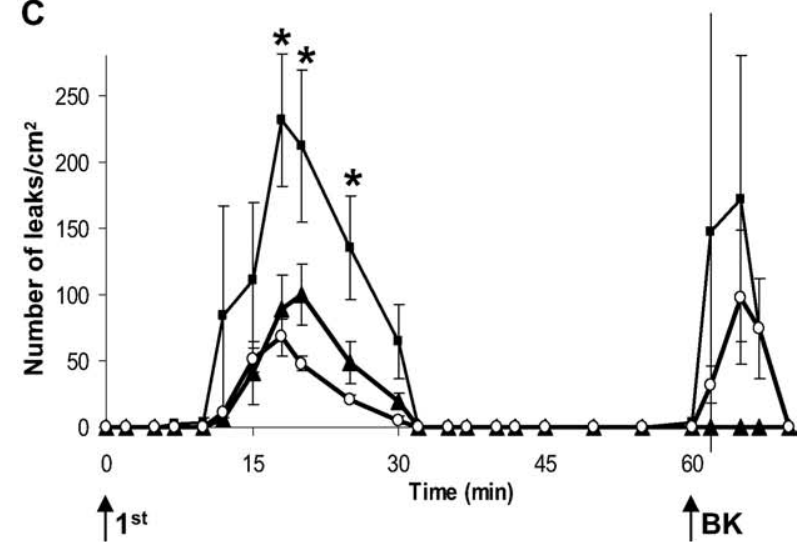

Fig. 1. Plasma leakage in the HCP induced by L. donovani and L. chagasi promastigotes was enhanced by the ACE inhibitor, captopril.

Three series of hamster experiments were carried out (A-C) in which each series consisted of three groups of animals $(N=5-11$ in each group and altogether 60 hamsters) divided into control (O), Cap- $(\mathbf{\square})$ and Cap+HOE140-pretreatment (A). In all three series, Cap or Cap+HOE-140 was applied for $5 \mathrm{~min}$ prior to the application of promastigotes or their homogenates $\left(0.5 \mathrm{ml}\right.$ of $2 \times 10^{8}$ per $\left.\mathrm{ml}\right)$ that remained on the HCP for $10 \mathrm{~min}$. At $60 \mathrm{~min}$, $\mathrm{BK}(250 \mathrm{nM})$ was applied for $5 \mathrm{~min}$. A. Mean number of postcapillary venular leaks following two topical applications of $10^{8}$ parasites (L. donovani) on the HCP with 30 min interval. B. Mean number of postcapillary venular leaks following two topical applications of pHMG (homogenates of $10^{8}$ parasites, L. donovani) on the HCP with $30 \mathrm{~min}$ interval. C. Mean number of postcapillary venular leaks following one topical application of $0.5 \mathrm{ml}$ of $2 \times 10^{7}$ per $\mathrm{ml}$ of $L$. chagasi metacyclic parasites. There were no significant $(P>0.05)$ differences between the responses to BK in captopril or control groups in any of the three series but HOE-140 completely blocked the effect of BK. (*) $=P<0.05$ as compared with control group. age response was observed after addition of $\mathrm{BK}$ at this time $\left(197 \pm 43\right.$ leaks per $\left.\mathrm{cm}^{2}, N=11\right)$, i.e. $60 \mathrm{~min}$ after the first and 30 min after the second application of promastigotes. In a separate series of experiments the response to BK at $30 \mathrm{~min}$ after the first parasite application was even larger $\left(257 \pm 35\right.$ leaks per $\left.\mathrm{cm}^{2}, N=10\right)$. These results suggested that the state of non-responsiveness to the second application of the parasites was not caused by $\mathrm{B}_{2} \mathrm{R}$ down-regulation. At the end of the Series I experiments, BK induced a prominent microvascular leakage, indicating that the $\mathrm{HCP}$ was functionally preserved (Fig. 1A).

In the Series II experiments, the Cap-HCP tissues were treated with L. chagasi promastigotes. Apart from belonging to a different Leishmania complex, the L. chagasi preparation used here (isolated from 10\% Ficoll gradient cushions) was enriched with complement resistant metacyclic promastigotes (Fig. 1C). Similar to data obtained with L. donovani complex, the maximal response in the Cap group $\left(231 \pm 50\right.$ leaks per $\left.\mathrm{cm}^{2}\right)$ was significantly $(P<0.05)$ larger than the response in the Cap + HOE-140 $(100 \pm 23$ leaks per $\left.\mathrm{cm}^{2}\right)$ and the control group $\left(68 \pm 14\right.$ leaks per $\left.\mathrm{cm}^{2}\right)$. Moreover, the venular leakage that $L$. chagasi evoked in Cap-HCP was reduced by HOE-140. Of note, the L. chagasi metacyclics provoked a larger and more lasting response in Cap$\mathrm{HCP}$, peaking at $18 \mathrm{~min}$ as compared to $15 \mathrm{~min}$ observed with L. donovani (Fig. 1C). Another difference with respect to $L$. donovani-induced responses was that $L$. chagasi metacyclics were able to provoke significant, albeit moderate $\mathrm{B}_{2} \mathrm{R}$ dependent leakage in the absence of Cap (data not shown). The reasons for the greater dependence of ACE inhibitor on L. donovani assays as opposed to those observed with L. chagasi are not known, but could relate to qualitative/quantitative differences in the expression of molecules that initiate inflammation by metacyclic versus stationary-phase promastigotes. As to the different kinetics observed with these two species, it is also possible that species specific differences in surface expression of hyaluran-binding proteins [34] may influence the parasite ability to move/migrate through the hyaluranrich extracellular matrix of the HCP.

The critical role of $\mathrm{B}_{2} \mathrm{R}$ in parasite-driven inflammation was further demonstrated by studying $L$. donovani-evoked edema in the mouse paw. As in the studies with the HCP, vigorous edematogenic responses were observed in $\mathrm{BALB} / \mathrm{c}$ (Fig. 2A) or J129 wild-type mice (Fig. 2B) pre-treated with the ACE inhibitor. The responses in Cap-treated BALB/c were blocked by HOE-140 while the promastigotes failed to evoke significant edematogenic responses in Cap- $\mathrm{B}_{2} \mathrm{R}\left(\digamma^{-}\right)$mice (Fig. 2B).

\subsection{Homogenates of promastigotes activate the kinin pathway in hamster and mice}

In a third series of experiments, we asked if infection was critically required for the induction of the microvascular responses induced by the parasites. We first approached this question by testing the effects of $L$. donovani homogenates 
A

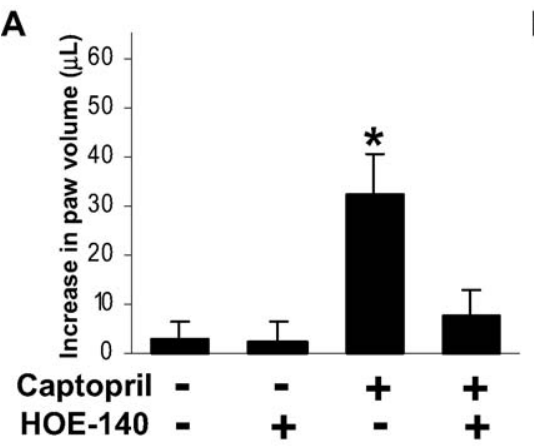

B

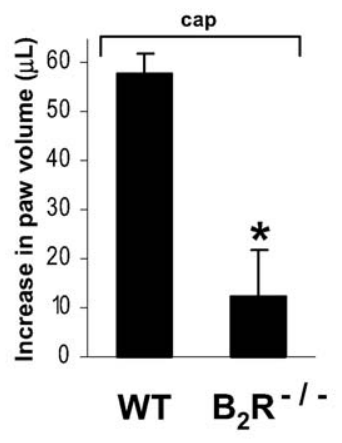

C

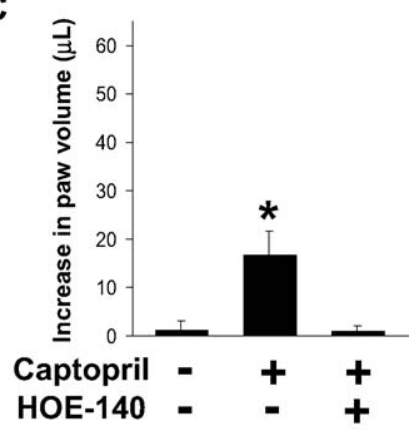

Fig. 2. Captopril potentiates $\mathrm{B}_{2} \mathrm{R}$-driven edema in mice injected with L. donovani.

A. Data represent mean values of differences between right and left paw volumes measured $3 \mathrm{~h}$ after inoculation of $10 \times 10^{6}$ promastigotes in the right paw of BALB/c mice while the contralateral paw was injected with PBS. Cap $(4 \mathrm{mg} / \mathrm{kg})$ was injected i.p. $1 \mathrm{~h}$ before parasite inoculation $(N=5)$, while a second group of mice received PBS as a control. The third group was pre-treated with Cap and HOE-140 (200_g/Kg). B. L. donovani promastigotes were inoculated in the paw of male wild-type (WT) $\mathrm{J} 129$, or male $\mathrm{J} 129 \mathrm{~B}_{2} \mathrm{R}^{-} /$mice. Both groups received Cap $(4 \mathrm{mg} / \mathrm{kg}) 1 \mathrm{~h}$ prior to infection. $(*)=P<0.05$. C. Edema $(3 \mathrm{~h})$ induced by pHMG (equivalent to $10 \times 10^{6} \mathrm{~L}$. donovani) inoculation in the mouse paw. Mice were pre-treated with Cap or HOE-140 as described above. Each group consisted of 5 mice. $(*)=P<0.05$

(pHMG) (Fig. 1B) — the dose used being equivalent to that of living promastigotes. Unlike the potent but protracted $\mathrm{B}_{2} \mathrm{R}$ driven leakage responses elicited by living parasites (see for comparison Cap-HCP, in Fig. 1A), the microvascular reaction induced by $\mathrm{pHMG} / \mathrm{Cap}$ was mild $(P<0.05)$ but evolved faster, peaking within 5-10 min of topical application (Group III.1), being extinguished more rapidly than observed with living parasites (Fig. 1A). It is noteworthy that Cap-HCP responded to the second (consecutive) application of $\mathrm{pHMG}$ (Fig. 1B), i.e. we did not observe the characteristic tachyphylaxis induced after the first stimulation by living promastigotes (see for comparison Fig. 1A). Interestingly, the macromolecular leakage elicited by the first application of $\mathrm{pHMG}$ did not significantly involve the kinin pathway (Fig. 1B) because the responses were only marginally increased by Cap (Group III.2) and HOE-140 did not significantly reduce these permeability changes $(P>0.05)$, irrespective of the presence or absence of Cap (Group III.3). Strikingly, however, addition of the ACE inhibitor significantly augmented the leakage induced by the second $\mathrm{pHMG}$ application, 30 min later $(P<0.05)$ (Fig. 1B) and this response was abrogated by HOE$140(P<0.05)$. Hence, our data suggest that the primary inflammatory response evoked by $\mathrm{pHMG}$ (i.e. driven by kininindependent pathways) has rendered the microvascular bed more sensitive to the endogenously released kinins generated upon the second $\mathrm{pHMG}$ challenge $\left(\mathrm{B}_{2} \mathrm{R}\right.$ dependent). Assays in the mouse model (paw edema) confirmed that $\mathrm{pHMG}$ induces permeability changes via $B_{2} R$ (Fig. $2 C$ ). Like the effects of living parasites, the edematogenic responses induced by $\mathrm{pHMG}$ are down-regulated by ACE.

\subsection{Activation of the kinin pathway in vivo depends on the activity of L. donovani cysteine proteinases}

As mentioned in the introduction, previous studies on T. cruzi trypomastigotes have linked the activation of the kinin system to the activity of cruzipain, the parasite's major cys- teine proteinase (CP; reviewed in [35]). Given indications that L. donovani promastigotes also express developmentally regulated cathepsin L-like CP [36], we first asked if L. donovani could rely on CP to activate the kinin system. We first evaluated if parasite pre-treatment with N-Pip-F-hF-VSPh (K11777) would impair their ability to activate the mouse kinin pathway in vivo. Indeed, the results (Fig. 3B) show that the edema evoked by K11777-treated promastigotes was significantly attenuated in Cap-BALB/c, as compared to responses induced by control parasites. Consistent with these results, an attenuated microvascular leakage response was observed when we applied K11777-treated promastigotes to Cap-HCP (Fig. 3A). Combined, these experiments suggest that $L$. donovani promastigotes share with $T$. cruzi the ability to activate the kinin pathway through cysteine proteinases. Further, the potentiation observed in Cap-treated animals implies that ACE down-modulates the otherwise robust inflammatory response that these pathogens can evoke in mammals. Consistent with these results, K11777-treated pHMG did not induce significant edema in Cap-mice (data not shown).

\subsection{L. donovani promastigotes liberate kinins from $\mathrm{HK}$ through the activity of cysteine proteinases}

Whether exposed at the cell surface or originating from extravasated plasma, kininogens must be properly processed in order to release kinins. In a previous study [23], we used Western blotting with mAb MBK3 to identify the bradykinin epitope (domain 4) in soluble HK molecules incubated with living T. cruzi trypomastigotes. Here we extended this analysis to L. donovani promastigotes. First, we checked if the parasites generated HK fragments, identified with a mAb (HKL13) directed against its light chain. The degradation profiles were too complex (data not shown), resembling of those described for HK processing by cruzipain [23]. Most importantly, however, was the profile observed with MBK3 (Fig. 4). 
A

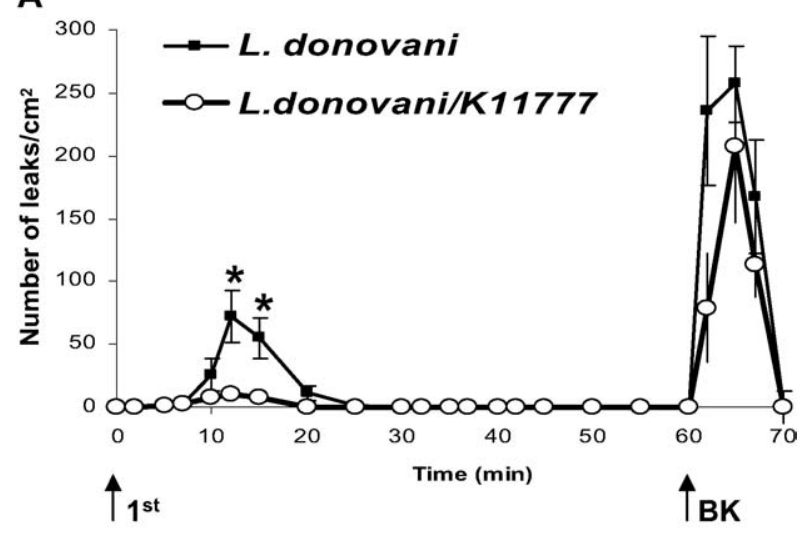

B

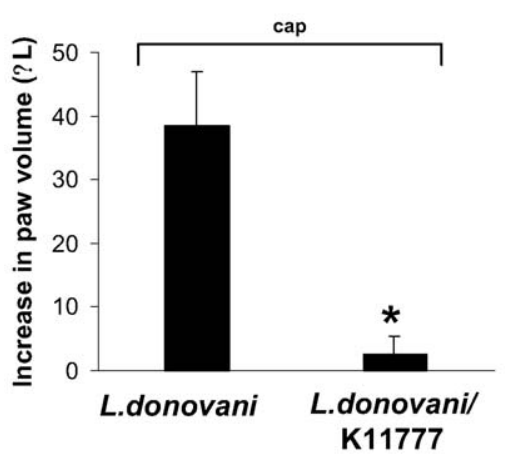

Fig. 3. Parasite cysteine proteinases are required for kinin pathway activation in vivo.

A. Comparison of permeability inducing properties of living L. donovani promastigotes pre-treated with $10 \mu \mathrm{M}$ of the irreversible cysteine proteinase inhibitor N-Pip-F-hF-VSPh (K11777) versus PBS/DMSO-treated controls. Data represent mean number of postcapillary venular leaks following two topical applications of parasites (control or pre-treated with K11777) at 0 and $30 \mathrm{~min}$ followed by one final application of BK $(250 \mathrm{nM})$ at $60 \mathrm{~min}$ (see arrow). The HCP was superfused with $1 \mu \mathrm{M}$ of Cap. $(*)=P<0.05$. B. Edema induced by paw inoculation of $L$. donovani promastigotes in Cap-treated BALB/c mice. The parasites were pre-treated with K11777, as described above.

First, our positive control shows that the BK epitope was lost when HK was treated with tissue kallikrein (Fig. 4, track e) or when it was incubated with $L$. donovani promastigotes (Fig. 4, track b). Interestingly, addition of N-Pip-F-hF-VSPh (K11777) to the parasite suspension partially inhibited the proteolytic excision of the BK epitope from intact HK molecules (Fig. 4, track c). As an additional control, the addition of EDTA, aimed at inhibiting metalloproteinases, such as Gp63 [37], had only marginal protective effects on the BK display (track d), thus ruling out an important role for these developmentally regulated metalloproteinases. Our data suggest that whether acting alone or in conjunction with other parasite proteinases, L. donovani cysteine proteinases may efficiently process $\mathrm{HK}$, the reaction being coupled to liberation of the bradykinin moiety. Assays performed with L. chagasi metacyclic promastigotes indicated that BK was also released from $\mathrm{HK}$, but at lower efficiency as compared to L. donovani (data not shown).

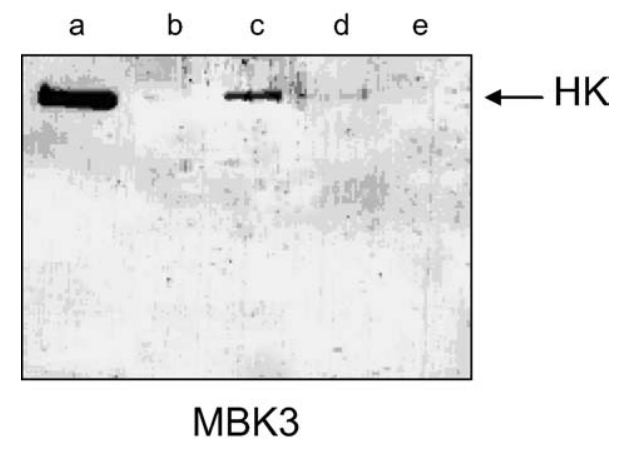

Fig. 4. Parasite-mediated processing of HK is cysteine proteinase dependent and promotes excision of the BK epitope. Stationary-phase L. donovani promastigotes were resuspended in HAM ( $\mathrm{pH} 6.5$ ) supplemented with $10 \mu \mathrm{g} / \mathrm{ml}$ of $\mathrm{HK}$ and incubated for $1 \mathrm{~h}$ at $26^{\circ} \mathrm{C}$ in the presence of DTT. Involvement of cysteine proteases and metalloproteinases were studied by adding $10 \mu \mathrm{M}$ of N-Pip-F-hF-VSPh (K11777) or $50 \mathrm{mM}$ of EDTA. At the end of the incubation period, parasite motility was the same as in untreated controls. The cellfree supernatants were boiled in SDS-2-ME lysis buffer. Western blotting carried out using mAb anti-BK (MBK3) Positive controls for proteolytic excision of BK were performed by mixing tissue kallikrein $(2 \mu \mathrm{g} / \mathrm{ml})$ with the HK sample. Arrow indicates position of intact HK chain $(118 \mathrm{kDa})$. Lanes (a), HK control; (b), HK + Promastigotes; (c), HK + N-Pip-F-hFVSPh-treated-promastigotes; (d), HK + EDTA-treated-promastigotes; (e), $\mathrm{HK}+\mathrm{TK}$

\subsection{Molecular characterization of kininogenases from L. donovani and L. chagasi}

Considering that L. donovani and L. chagasi cysteine proteinases are sensitive to N-Pip-F-hF-VSPh (K11777), we set out to investigate their biochemical properties in NP-40 lysates from L. donovani and L. chagasi promastigotes. In both species, we detected E-64 and K11777-sensitive peptidases in either (i) living parasites (Fig. 5A) (ii) culture supernatants (Fig. 7B) and (iii) NP-40 lysates (Fig. 5B; for brevity, only the data with $L$. donovani is illustrated). The active enzymes present in NP-40 lysates were characterized by affinitylabeling the $L$. donovani cysteine proteinase(s) with biotinN-Pip-urea-F-hF-VSPh. As specificity controls, we preincubated the lysates with E-64, prior to treatment with the biotinylated N-Pip-urea-F-hF-VSPh probe. The biotinylated reaction products (Fig. 5C) identified papain-like enzymes of $35-40 \mathrm{kDa}$ as the L. donovani targets of N-Pip-urea-F-hFVSP.

\subsection{Activation of the kinin pathway modulates the uptake of promastigotes by macrophages}

Earlier work with $T$. cruzi $[21,23]$ suggested that cruzipainmediated proteolysis of HK may occur when the kinin precursor proteins bind to heparan sulfate or other surface binding sites on endothelial cells $[38,39]$. Initial studies with $L$. donovani suggested that similar mechanisms underlie parasite uptake by mouse peritoneal inflammatory macrophages (P.R. Batista and J. Scharfstein, data not shown). Encouraged by these preliminary data, we then examined if HK could serve as kinin precursors for L. chagasi $\mathrm{CP}$ during the patho- 
A

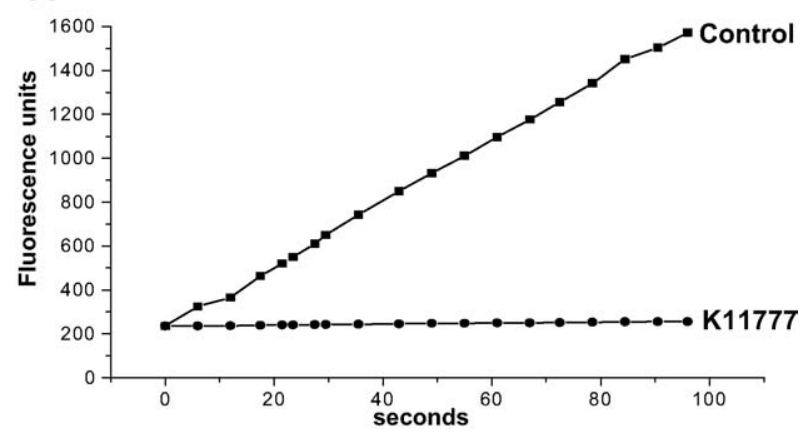

B

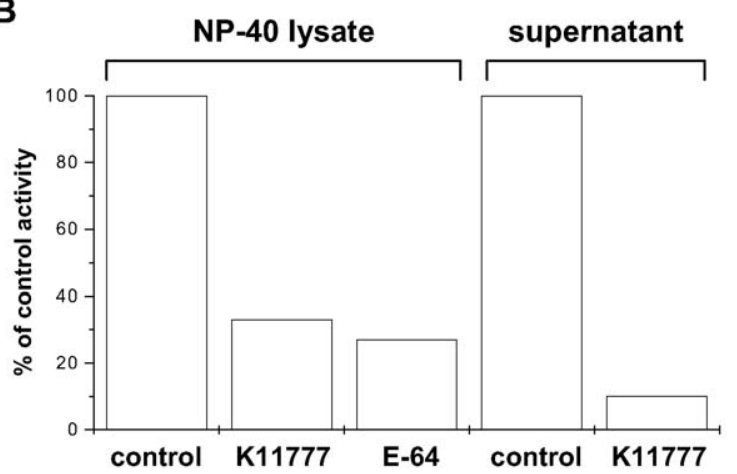

C

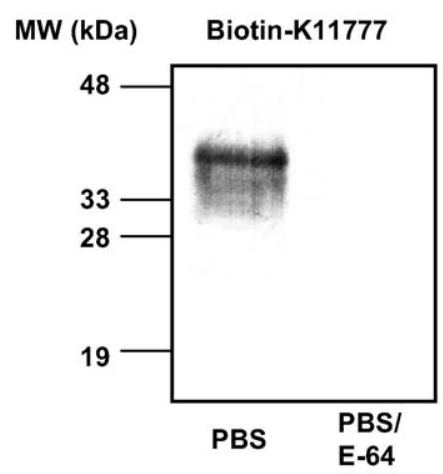

Fig. 5. Molecular characterization of $L$. donovani cysteine protease(s). (A) Promastigotes obtained after 7 days of culture were lyzed in PBS-1\% NP-40 on ice. The soluble fraction was recovered by centrifugation and the peptidase activity of the lysates $(1 \mu \mathrm{g} / \mathrm{ml})$ was monitored by the hydrolysis of CBZ-Phe-Arg-MCA. (A) The plot shows the increase of fluorescence with time, reflecting product formation. The synthetic cysteine protease inhibitor N-Pip-F-hF-VSPh (K11777) was added at a $10 \mu \mathrm{M}$ final concentration. (B) The peptidase activity contained in lysates and in culture supernatants was monitored as described in A. E-64 and N-Pip-F-hF-VSPh were added at $30 \mu \mathrm{M}$ and $10 \mu \mathrm{M}$ final concentration, respectively. The $\%$ of inhibition was determined by comparing the initial velocities (as described in Section 2), where control was estimated as $100 \%$. (C) Active-site labeling of cysteine proteases was accomplished by treating parasite lysates $(30 \mu \mathrm{g})$ with $10 \mu \mathrm{M}$ of biotin-coupled N-Pip-F-urea-hF-VSPh for $1 \mathrm{~h}$. RT (lane 1); or in the same buffer supplemented with $30 \mu \mathrm{M}$ of E-64, prior to the addition of the biotinylated probe (lane 2). The samples were separated by SDS-PAGE, blotted onto nitrocellulose and the reactive products were visualized upon treatment with avidin-alkaline phosphatase.

gen's interaction with macrophages. In the first series of experiments (Fig. 6A-C), we determined the extent of parasite uptake after $4 \mathrm{~h}$ interaction with spleen adherent cells
(59\% CD11b positive) isolated from BALB/c mice. Addition of BK, over a range of concentrations (5-300 nM) to CapRPMI medium (serum-free), modulated parasite uptake by the spleen adherent cells, yielding a bell-shaped dose-effect profile (Fig. 6A). At low concentrations of BK, the number of internalized amastigotes gradually increased, peaking at $10 \mathrm{nM}$ BK (Fig. 6A) $(P<0.001)$. In contrast, adherent spleen cells treated with high-doses of BK (100-300 nM) showed reduced numbers of internalized parasites $(P<0.001)$, most likely reflecting down-regulation of $\mathrm{B}_{2} \mathrm{R}$ by agonist excess (Fig. 6A). The same bell-shaped profile was also observed when the effect was calculated as the percentage of cells infected (data not shown). Given indications that BK modulates promastigote uptake by the mouse spleen adherent cells in a dose-dependent manner, we then asked if purified (human) HK could serve as an exogenous source of kinin precursor protein, the underlying premise being that the parasites would rely on $\mathrm{CP}$ to liberate the $\mathrm{B}_{2} \mathrm{R}$ kinin agonist, as suggested by previously presented data (Figs. 3 and 4). Indeed, the results showed that $\mathrm{HK}(5-300 \mathrm{nM})$ induced dramatic changes in parasite uptake by spleen adherent cells (Fig. 6B). As in the bell-shaped profile induced by BK, addition of excess HK (>100 nM) to Cap-medium reduced parasite uptake to baseline levels $(P<0.001)$. Peak values for HK were observed at slightly higher concentration $(30 \mathrm{nM})(P<0.001)$ than BK, suggesting that about $30 \%$ of the exogenously supplied HK proteins were quantitatively converted into $\mathrm{B}_{2} \mathrm{R}$ agonists during the $4 \mathrm{~h}$ period of host-parasite interaction (Fig. 6B). Further, the stimulation of parasite uptake observed at the optimal $(30 \mathrm{nM})$ dose of HK was abolished by HOE140 (Fig. 6C). These results suggested that HK modulated parasite uptake by mouse spleen adherent cells via release of the $\mathrm{B}_{2} \mathrm{R}$ agonist, rather than acting merely as cell adhesive molecules [40]. To verify if the $B_{2} R$ agonist was released from $\mathrm{HK}$ (tested at $30 \mathrm{nM}$ ) via parasite cysteine proteinases, we added the CP inhibitor K11777 $(10 \mu \mathrm{M})$ to the Cap-RPMI medium. The results (Fig. 6C) show that uptake of K11777treated promastigotes by mouse adherent spleen cells was significantly reduced $(P<0.01)$, and to the same extent as the $\mathrm{B}_{2} \mathrm{R}$ antagonist, HOE-140. Furthermore, the combination of K11777 and HOE-140 did not further attenuate parasite uptake by the spleen adherent cells (Fig. 6C), suggesting that $\mathrm{HK}$ and $\mathrm{CP}$ are components of the same activation pathway. We then checked if these findings were also applicable to spleen adherent cells, isolated from the hamster. Addition of Cap alone to the hamster adherent cells (i.e. in the absence of exogenous BK or HK) led to a slight increase $(P<0.05)$ in parasite uptake (Fig. 6D). Albeit mild, this enhancement was abolished by HOE-140 (Fig. 6D). These results suggest that endogenous kininogens, either adsorbed from bovine serum on cell surfaces [23] or synthesized de novo by the hamster spleen cells, may have served as "endogenous" kinin precursor molecules. Consistent with data shown for mice spleen adherent cells (Fig. 6A), addition of $50 \mathrm{nM} \mathrm{BK}$ to Capmedium further increased parasite uptake by hamster spleen cells, whereas HOE-140 antagonized these effects $(P<0.05)$ 
A

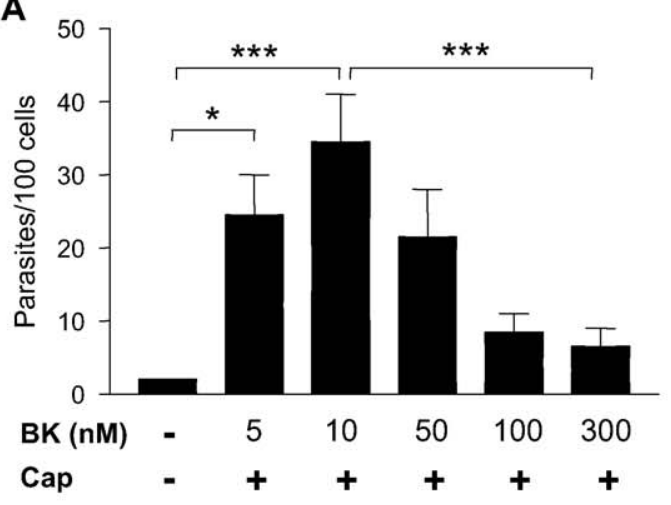

C

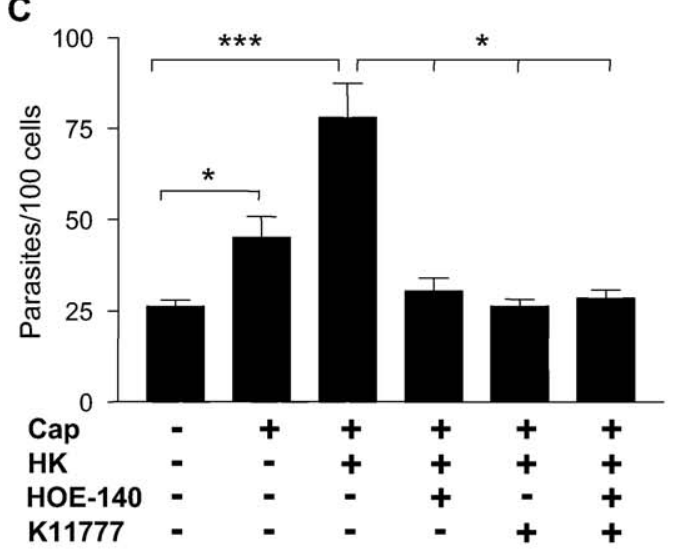

E

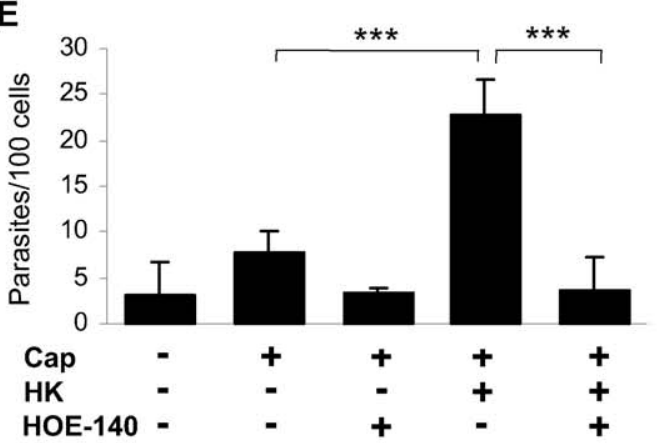

B

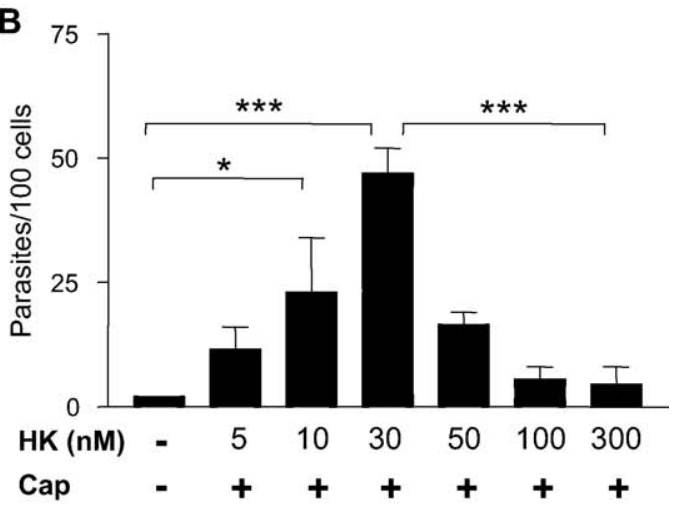

D

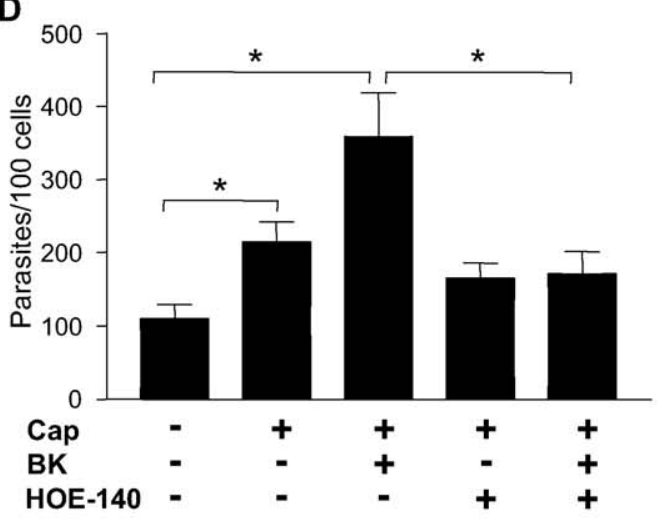

F

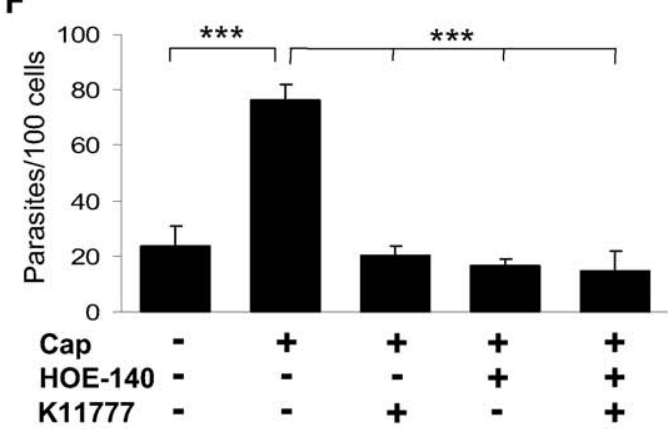

Fig. 6. Activation of $B_{2} R$ modulates uptake of promastigotes by macrophages. A. Dose-dependent effects of exogenous BK. Mouse (BALB/c) spleen adherent cells were incubated with Cap $(25 \mu \mathrm{M})$ in serum-free RPMI supplemented with BK (5-300 nM) 5 min before addition of Leishmania promastigotes (parasite:macrophage ratio of 5:1). The interaction proceeded for $4 \mathrm{~h}$ in RPMI. B. Dose-dependent modulation of parasite uptake by purified HK. Same cells as described above, using HK (5-300 nM) and Cap. C. Interplay of cysteine proteinases, ACE and HK in the modulation of promastigote uptake by adherent spleen cells. Interaction was performed in serum-free RPMI, as described above, in the presence or absence of HK (30 nM), Cap (25 $\mu$ M), HOE-140 (100 nM), and/or the cysteine protease inhibitor K11777 $(10 \mu \mathrm{M})$. D. HK modulates uptake of promastigotes by hamster spleen adherent cells. Interaction of hamster spleen adherent cells with $L$. chagasi promastigotes (5:1) was carried out for $4 \mathrm{~h}$ in serum-free RPMI supplemented with BK ( $30 \mathrm{nM})$. Where indicated, HOE- 140 (100 nM) was added to Cap-medium. E. Activation of $\mathrm{B}_{2} \mathrm{R}$ stimulates uptake of promastigotes by mouse resident peritoneal macrophages. Promastigotes were incubated with $\mathrm{BALB} / \mathrm{c}$ resident peritoneal macrophages for $4 \mathrm{~h}$ in serum-free medium supplemented, or not, with Cap $(25 \mu \mathrm{M}), \mathrm{HK}(30 \mathrm{nM})$ and/or HOE-140 (100 nM). F. Interplay between parasite cysteine proteinases, serum-bovine kininogens and ACE influences the extent of parasite uptake by mouse resident peritoneal macrophages. Interaction with resident peritoneal macrophages was performed as described above, except for the presence of $10 \%$ FCS in the RPMI medium. The influence of parasite CP was tested by pre-incubating $(10 \mathrm{~min})$ stationary-phase promastigotes with $\mathrm{K} 11777(10 \mu \mathrm{M})$. After removing the excess of K11777 by washing, the K11777-treated promastigotes were added to the interaction medium, in the presence or absence of Cap and/or HOE-140. Data values for $\%$ infected cells (not shown) matched number of parasites per 100 host cells. $(*) P<0.05,(* *) P<0.01,(* * *) P<0.001$ for difference between groups.

(Fig. 6D). In short, these data suggest that, irrespective of the origin of spleen adherent cells, i.e. mice or hamsters, parasiteinduced activation of $\mathrm{B}_{2} \mathrm{R}$ stimulates pathogen uptake by spleen adherent cells. Of note, the potentiating effects of Cap suggest that this kinin-driven cellular response is tightly regulated by ACE.

Given that spleen cells contain a heterogeneous population of macrophages, the above experiments were repeated 

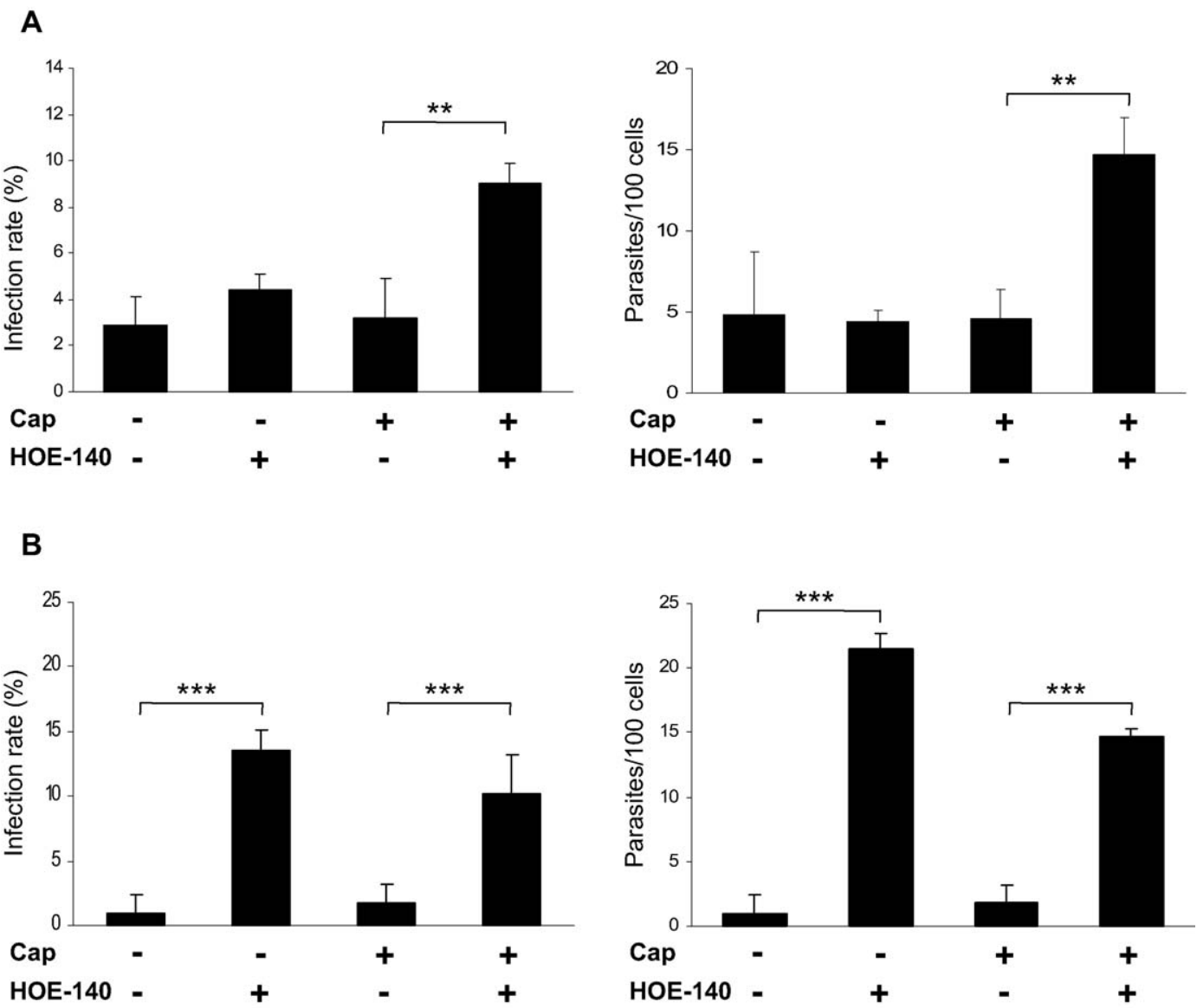

Fig. 7. Intracellular amastigote outgrowth in resident or TG-induced macrophages is differentially modulated by $A C E$ and $B_{2} R$. $A$, kinins and $A C E$ modulate amastigote outgrowth in resident peritoneal macrophages.

The $4 \mathrm{~h}$ host-parasite interaction was carried out in RPMI-10\% FCS, supplemented or not with Cap and/or HOE-140. At the end of this period (4 h), the parasites remaining in supernatants were removed, the wells were washed, and fresh RPMI-FCS was added for another $72 \mathrm{~h}$. B, $\mathrm{B}_{2} \mathrm{R}$ modulates amastigote outgrowth in TG-induced macrophages. Interaction between host and parasites were carried out in RPMI-FCS as described above. Results are expressed either as $\%$ infected cells (left panel) or number of intracellular amastigotes $/ 100$ macrophages (right panel). $(*) P<0.05,(* *) P<0.01,(* * *) P<0.001$ for difference between groups.

with mouse peritoneal macrophages. As in the previous assays, the interaction with resident peritoneal macrophages (BALB/c) was initially performed in serum-free RPMI medium. Addition of Cap alone had little effect on parasite uptake by resident macrophages. However, the parasite internalization was greatly enhanced $(P<0.001)$ upon addition of purified HK to the Cap-treated cultures (Fig. 6E). We noted that the baseline levels of parasite uptake observed in the absence of Cap (see left bar, Fig. 6E) were far lower than expected for fully functional phagocytic cells. This raised concerns that serum-deprivation, during the host-parasite interaction period $(4 \mathrm{~h})$, had decreased the phagocytic activity and, possibly, other key functions of mouse peritoneal macrophages. To eliminate this potential artifact, macrophageparasite interactions were then exclusively carried out in medium containing RPMI-10\% FCS. As anticipated, baseline levels of parasite uptake by the macrophages were now markedly increased (about eightfold, see Fig. 6F, control bar) as compared to the baseline values observed in the absence of serum (Fig. 6E). The addition of the ACE inhibitor again stimulated increase in parasite uptake $(P<0.001)$ (Fig. 6E).
Furthermore, the Cap-driven potentiation of parasite uptake by resident macrophages was blocked by HOE-140, further implicating the $\mathrm{B}_{2} \mathrm{R}$ activation pathway in this process (Fig. 6F). In the same set of experiments, we again used K11777 to verify if the activity of parasite-derived CP was required for $\mathrm{B}_{2} \mathrm{R}$-driven uptake of parasites in RPMI-10\% FCS. To eliminate risks of non-specific effects of K11777 on macrophage $\mathrm{CP}$, the promastigotes were pre-treated for $10 \mathrm{~min}$ with K11777. After removing the excess of irreversible CP inhibitor from the cultures, we added K11777-treated promastigotes to the RPMI-FCS cultures. The data revealed that macrophages did not efficiently internalize K11777promastigotes (Fig. 6E). These data suggest that generation of $\mathrm{B}_{2} \mathrm{R}$ agonists in the cultures may depend on parasite $\mathrm{CP}$-mediated processing of bovine serum kininogens.

\subsection{Parasite survival in resident and TG-macrophages is differentially modulated by $B_{2} R$ and $A C E$}

After demonstrating that kinin pathway activation modulates promastigote uptake by resident macrophages, we stud- 
ied the fate of the internalized parasites, $72 \mathrm{~h}$ later. In this set of experiments, promastigotes were incubated with macrophages (4 h) in RPMI-10\% FCS, in the presence (or not) of Cap and/or HOE-140. At the end of this period, the cultures were washed, and then incubated with fresh RPMI-FCS for another 72 h. Our results (Fig. 7) indicate that the increased parasite uptake observed after $4 \mathrm{~h}$ of cellular interaction in Capmedium (RPMI-FCS) (Fig. 6F) did not translate into increased parasite survival (Fig. 7A), whether measured as percentage of infected cells (Fig. 7B, left panel) or number of intracellular amastigotes/100 cells (Fig. 7B, right panel). The presence of HOE-140 alone in the cellular interaction (4 h) medium (RPMI-10\%FCS) did not stimulate parasite survival, $72 \mathrm{~h}$ post-infection. Surprisingly, however, a vigorous increase (19 fold) of intracellular amastigotes was observed in cultures ( $72 \mathrm{~h}$ post-infection) that had been treated, during the $4 \mathrm{~h}$ interaction period, with both Cap and HOE-140 $(P<0.05)$ (Fig. 7A). The finding of this prominent amastigote burden at $72 \mathrm{~h}$ post-infection indicates that blockade of $\mathrm{B}_{2} \mathrm{R}$ signaling and ACE activity during the $4 \mathrm{~h}$ interaction period has converted resident macrophages into more susceptible hosts for L. chagasi. In other words, the combined action of HOE140 and Cap on resident macrophages suppressed the host protective responses that, in normal cells, efficiently constrain intracellular amastigote outgrowth.

We then asked if activation of the kinin system modulated parasite survival in inflammatory macrophages. Strikingly, the results showed that here, presence of HOE-140 during the $4 \mathrm{~h}$ interaction period vigorously stimulated parasite survival (72 h post-infection) in TG-macrophages (Fig. 7B, left and right panels). Importantly, the ACE inhibitor was not required here, indicating that HOE-140 mediated blockade of $\mathrm{B}_{2} \mathrm{R}$ signaling was sufficient to suppress the innate responses of TG-macrophages, converting them into highly susceptible host cells.

\section{Discussion}

This study was initiated because the innate immune system is known to be modulated by kinin peptides [13] and its principal aim was to establish if the kinin pathway was activated by the Leishmania species that cause visceral leishmaniasis. The choice of the hamster as one of the animal models was based on the fact that this particular species reproduces the pathological manifestations of visceral leishmaniasis observed in humans. Initially, our intravital microscopy studies showed that in the absence of ACE inhibitors, the L. donovani promastigotes evoked moderate but significant microvascular leakage, via kinin-independent pathways. L. chagasi metacyclics also induced moderate leakage in absence of Cap, but this response was abolished by HOE-140 (data not shown). We are currently studying if differences in parasite-induced activation of complement $[2,4,41]$ or production of PAF [42] may influence the extent of kinin system activation. In order to improve the detection of short-lived kinin peptides, pro- duced in tissues exposed to promastigotes, we used a strategy that had proved rewarding in our previous studies of endothelial interactions with $T$. cruzi $[21,23,24]$. This involved blockade of kinin-degradation pathways by pre-treating the $\mathrm{HCP}$ or mice with ACE inhibitor, prior to challenge with parasites. Pharmacological interventions, combined with the use of $\mathrm{B}_{2} \mathrm{R}^{-}{ }^{-}$mice, showed that the macromolecular leakage (hamster) and edematogenic inflammation induced by $L$. donovani or L. chagasi promastigotes in animals pre-treated with Cap depended on activation of $\mathrm{B}_{2} \mathrm{R}$ by endogenously released bradykinin or lysyl-bradykinin. Time course studies revealed that the $\mathrm{B}_{2} \mathrm{R}$-dependent leakage induced by promastigotes evolved rather slowly in the Cap-HCP. The response was clear by $10 \mathrm{~min}$ after parasite application, it peaked at 15 min and decreased sharply thereafter. These temporal dynamics contrasted with the immediate response induced by topical application of histamine or bradykinin, which typically peaked after $2 \mathrm{~min}$, but not later than $5 \mathrm{~min}[43,44]$, and were also different from the vascular leakage observed after ischemia/reperfusion (peak value at $10 \mathrm{~min}$ of reperfusion), a process caused by activated leukocytes [45]. Our finding that L. chagasi promastigotes elicited a somewhat delayed response (peaking at $18 \mathrm{~min}$ ) as compared to L. donovani (15 min) is intriguing, in view of the overall similarities of the activation profile of these species. The reasons for this difference in kinetics are not known, but could possibly be related to differential ability of these flagellates to move or migrate through the hyaluran-rich extracellular matrix of the HCP [34].

Although not quantified here, we have consistently observed increased rolling/adhesion of leukocytes in postcapillary venules soon after promastigote application. This is of interest in the light of studies in the rat mesentery showing that application of high-doses of synthetic BK caused leukocyte rolling/adhesion to the endothelium by a P-selectin and ICAM-1 dependent pathway, due to the action of oxidative products generated via cytochrome P-450 [46]. Interestingly, recent observations made in a mouse airpouch model indicate that Leishmania-induced neutrophil migration within a few hours, the cellular responses being coupled with formation of pro-inflammatory cytokines (TNF- $\alpha$ and IL-1 $\beta$ ) [47]. At the present time, the nature of the mediators that induce early leukocyte-endothelium adherence in Cap-HCP exposed to $L$. donovani or L. chagasi promastigotes is unknown. Rather than kinins, inflammation evoked by promastigotes of the L. donovani complex may also depend on pattern recognition by innate immunity receptors, such as those from the Toll-like receptor (TLR) family [48]. The complement system is another pathway worth considering in this context, because there is evidence that complement anaphylatoxins induce microvascular leakage in the cheek pouch [49]. In addition, activation of the complement system was previously implicated in the acute inflammatory process, which L. chagasi promastigotes induced in hamsters [41]. Hence, whether induced by anaphylatoxins, by TLR-dependent activation of leukocytes [48], or by other as yet uncharacterized activation 
mechanisms, impairment of the integrity of the endothelial barrier at very early stages of inflammation elicited by L. chagasi or L. donovani may allow efflux of plasma proteins, including kininogens, into extravascular infection sites. Acting further downstream, parasite proteases may then process the rapidly accumulating kinin precursor proteins, liberating high levels of kinins that, acting through $B_{2} R$, further amplify the initial inflammatory reaction.

Studies with homogenates of promastigotes offered clues to the molecular basis of this process. First, we found that the kinin system can be activated without need of active infection. Assays with parasite homogenates (pHMG) indicated that diffusible pro-inflammatory molecules are entrapped within dead/disrupted parasite cells. pHMG evoked rapid macromolecular leakage (peaking at $10 \mathrm{~min}$, Fig. 1B), a feature that contrasts to the relatively protracted $(15 \mathrm{~min})$ permeability responses induced by living Leishmania flagellates. Of note, HOE-140 did not block the microvascular leakage elicited by the primary $\mathrm{pHMG}$ provocation, suggesting that inflammation is initiated by a kinin-independent route. By contrast, the $\mathrm{B}_{2} \mathrm{R}$ antagonist efficiently blocked the leakage response elicited by the subsequent pHMG challenge. These results suggest that activation responses elicited by the first pHMG provocation may have rendered the pouch's microvascular bed hypersensitive to the $\mathrm{B}_{2} \mathrm{R}$ agonists, formed by the second application of the homogenates. As already discussed, it is possible that the plasma leakage induced by the first pHMG stimulus led to accumulation of blood-borne kininogens, the kinin precursor proteins, into to extravascular tissues. Upon second provocation, the $\mathrm{CP}$ entrapped within pHMG may then generate kinins, activating the endothelium via $B_{2} R$.

Assays performed with the cysteine proteinase inhibitor N-Pip-F-hF-VSPh (K11777) [28] suggested that promastigote-derived $\mathrm{CP}$ participate in the molecular mechanisms underlying kinin system activation in vivo (i.e. mouse model of inflammation in the paw and microvascular leakage in hamster cheek pouch) and in vitro (parasite uptake by macrophages). It remains to be determined if this process is facilitated by other peptidases, either from parasite or host origin. However, immunochemical studies revealed that K11777 could inhibit $L$. donovani-mediated processing of purified HK, an enzymatic reaction that is coupled to excision of the kinin moiety from soluble HK. Affinity-labeling of parasite lysates with biotin-N-Pip-F-hF-VSPh characterized these $\mathrm{CP}$ as enzymes with apparent molecular weights of 35-45 kDa. These values are lower than predicted for the full length product of cathepsin L-like genes [36], most likely because they correspond to mature enzymes that have lost their proteolyticsensitive C-terminal extension, as reported for other type $1 \mathrm{CP}$ described in various pathogenic trypanosomatids [50]. Systematic analysis of the substrate specificity of individual recombinant $\mathrm{CP}$ isoforms from $L$. chagasi and $L$. donovani promastigotes is required to characterize the molecular mechanisms underlying the kinin-release reaction. The possibility that the kinin-releasing $\mathrm{CP}$ activity of promastigotes may be upregulated by extracellular matrix constituents (e.g. hyaluran) is worth considering, in view of previous studies with $T$. cruzi showing that heparan sulfate proteoglycans positively modulate cruzipain-mediated processing of HK [23]. It was suggested that the activity of cystatin-like domains of $\mathrm{HK}$ (which otherwise function as potent CP inhibitors) is constrained as result of mutual cooperative interactions of heparan sulfate chains with both HK and cruzipain, the end result being increased liberation of bioactive kinins [50].

As a surface peptidase expressed by macrophages [51], ACE (CD143) may also modulate the outcome of kinindriven interactions with L. chagasi. Indeed, assays of parasite uptake indicated that ACE down-modulates the macrophage responses which kinins otherwise convey via $\mathrm{B}_{2} \mathrm{R}$. First, we showed that addition of exogenous BK or HK to serumfree (Cap-treated) macrophage cultures modulated parasite uptake in a dose-dependent manner, due to the activation of $\mathrm{B}_{2} \mathrm{R}$. The bell-shaped dose-effect profiles induced by $\mathrm{BK}$ were rather similar to the previously reported $\mathrm{B}_{2} \mathrm{R}$-driven potentiation of $T$. cruzi infectivity of non-phagocytic cells $([21,24]$ Moreover, the findings that macrophages exposed to K11777-treated promastigotes did not efficiently internalize these pathogens further suggest that the catalytic activity of L. chagasi $\mathrm{CP}$ is required for parasite-mediated generation of $\mathrm{B}_{2} \mathrm{R}$ agonists.

It is noteworthy that in all the in vitro systems tested, irrespective of (i) presence or absence of serum in the interaction medium (ii) species (hamster or mouse) or (iii) tissue origin of macrophages (peritoneal versus spleen), the parasite uptake was drastically enhanced upon addition of ACE inhibitor (Cap) to the interaction medium. Further, HOE-140 consistently antagonized the potentiating effects which Cap induce on parasite uptake, thus implicating the $\mathrm{B}_{2} \mathrm{R}$ signaling pathway as the transducer of this innate effector response in macrophages. Additional studies are required to determine if activation of kinin/ $\mathrm{B}_{2} \mathrm{R}$ signaling pathway exerts this role by upregulating surface expression and/or activity of phagocytic receptors such as mannosyl/fucosyl receptors, CR3 $[3,52]$ or via scavenger receptors. Alternatively, kinins may stimulate active cellular invasion, perhaps engaging the $\mathrm{B}_{2} \mathrm{R} /\left[\mathrm{Ca}^{2+}\right]_{\mathrm{i}}$-dependent mechanism which drive $T$. cruzi infection of endothelial cells and cardiomyocytes $[21,24]$.

The fate of the promastigotes that were internalized via the kinin/ $\mathrm{B}_{2} \mathrm{R}$-dependent and independent routes was also investigated in our experiments. We performed these studies with resident peritoneal macrophages rather than spleen adherent cells, thus avoiding uncertainties related to heterogeneity of the mouse or hamster spleen macrophage population. Moreover, the interaction between parasites and peritoneal macrophages ( $4 \mathrm{~h}$ ) was performed in RPMI-(10\%) FCS, rather than in serum-free medium, to exclude stress-related effects caused by serum deprivation. Our results showed that the increased uptake of promastigotes by resident macrophages, i.e. the Cap-induced effects, resulting from increased activation of $\mathrm{B}_{2} \mathrm{R}$ (see effect of ACE inhibitor; Fig. 6D) did not translate into increased amastigote survival (assessed $72 \mathrm{~h}$ 
after initial host-parasite interaction, see Fig. 7A). On the contrary, the numbers of amastigotes (or \% infected macrophages) found in Cap-cultures were as low as those of control macrophage cultures (Fig. 6D), notwithstanding the fact that these macrophages had initially internalized high numbers of promastigotes (Fig. 6F). Intriguingly, the addition of HOE140 to the Cap-treated cultures drastically increased parasite survival at $72 \mathrm{~h}$ (Fig. 7A, B). Considering that the low parasite uptake observed at the end of the $4 \mathrm{~h}$ interaction period (see data for HOE-140 + Cap, Fig. 6F), the finding of increased parasite burden at $72 \mathrm{~h}$ post-infection suggests that early blockade of $\mathrm{B}_{2} \mathrm{R}$, when combined with $\mathrm{ACE}$ inhibition, converts the relatively resistant, resident macrophage into a highly susceptible host cell. Thus, the effects of the ACE inhibitor on the host-parasite interactions introduced a bias in the innate response of resident macrophages, somehow linking $\mathrm{ACE}$ function to regulation of the kinin/ $\mathrm{B}_{2} \mathrm{R}$ activation pathway.

In contrast to the above studies, analysis of inflammatory (TG-elicited) macrophages revealed that ACE inhibition not necessary for the kinin/ $\mathrm{B}_{2} \mathrm{R}$-dependent control of intracellular parasite outgrowth. Here, parasite survival was drastically increased in cultures supplemented with HOE-140, irrespective of presence or absence of Cap. These data suggest that $\mathrm{B}_{2} \mathrm{R}$ on TG-macrophages may have a lower activation threshold for kinins. Although not investigated here, it will be worth determining if the kinin/ $\mathrm{B}_{2} \mathrm{R}$ signaling pathway may negatively modulate mechanisms underlying TGF- $\beta$ production by macrophages. This possibility is intriguing, in light of recent evidence identifying $L$. chagasi-derived CP as enzymes that convert latent TGF- $\beta$ into active TGF- $\beta$ [53], a suppressor of macrophage activation.

In summary, our study demonstrates that activation of the kinin system by L. chagasi and L. donovani modulated inflammation and upregulated macrophage effector responses. Additional studies are required to determine if comparable changes in the kinin system can modulate innate and/or adaptive immunity in the more complex setting of Leishmania infection transmitted by sand-flies, the natural vector.

\section{Acknowledgements}

We wish to thank Carlos V.P. Barbosa for preparation of parasites for intravital microscopy experiments and FACS analysis, Angela Alves and Leila Faustino for technical assistance and Professor Y.S. Bakhle for constructive comments. This work was supported by CNPq/Universal, FAPERJ and by Volkswagen Stiftung.

\section{References}

[1] J.M. Ribeiro, Role of saliva in blood-feeding by arthropods, Annu. Rev. Entomol. 32 (1987) 463-478.
[2] L.A. Rosenthal, F.S. Sutterwala, M.E. Kehrli, D.M. Mosser, Leishmania major-human macrophage interactions: cooperation between Mac-1 (CD11b/CD18) and complement receptor type 1 (CD35) in promastigote adhesion, Infect. Immun. 64 (1996) 2206-2215.

[3] M.E. Wilson, R.D. Pearson, Roles of CR3 and mannose receptors in the attachment and ingestion of Leishmania donovani by human mononuclear phagocytes, Infect. Immun. 56 (1988) 363-369.

[4] M. Dominguez, I. Moreno, M. Lopez-Trascasa, A. Torano, Complement interaction with trypanosomatid promastigotes in normal human serum, J. Exp. Med. 195 (2002) 451-459.

[5] Y. Belkaid, S. Kamhawi, G. Modi, J. Valenzuela, N. Noben-Trauth, E. Rowton, J. Ribeiro, D.L. Sacks, Development of a natural model of cutaneous leishmaniasis: powerful effects of vector saliva and saliva preexposure on the long-term outcome of Leishmania major infection in the mouse ear dermis, J. Exp. Med. 188 (1998) 1941-1953.

[6] R.B. Gomes, C. Brodskyn, C.I. De Oliveira, J. Costa, J.C. Miranda, A. Caldas, J.G. Valenzuela, M. Barral-Netto, A. Barral, Seroconversion against Lutzomyia longipalpis saliva concurrent with the development of anti-Leishmania chagasi delayed-type hypersensitivity, J. Infect. Dis. 186 (2002) 1530-1534.

[7] Y. Belkaid, J.G. Valenzuela, S. Kamhawi, E. Rowton, D.L. Sacks, J.M. Ribeiro, Delayed-type hypersensitivity to Phlebotomus papatasi sand fly bite: An adaptive response induced by the fly? Proc. Natl. Acad. Sci. USA 97 (2000) 6704-6709.

[8] A.M. Bittencourt, Leishmaniasis, p. 597-651, in: S.G.D.W. (Ed.), Tropical Pathology, second ed, vol. 8, Springer, Berlin, 1995.

[9] C.R. Engwerda, M. Ato, P.M. Kaye, Macrophages, pathology and parasite persistence in experimental visceral leishmaniasis, Trends Parasitol. 20 (2004) 524-530.

[10] P.M. Gorak, C.R. Engwerda, P.M. Kaye, Dendritic cells, but not macrophages, produce IL-12 immediately following Leishmania donovani infection, Eur. J. Immunol. 28 (1998) 687-695.

[11] S. Ahmed, M. Colmenares, L. Soong, K. Goldsmith-Pestana, L. Munstermann, R. Molina, D. McMahon-Pratt, Intradermal infection model for pathogenesis and vaccine studies of murine visceral leishmaniasis, Infect. Immun. 71 (2003) 401-410.

[12] K.D. Bhoola, C.D. Figueroa, K. Worthy, Bioregulation of kinins: kallikreins, kininogens, and kininases, Pharmacol. Rev. 44 (1992) $1-80$.

[13] J. Aliberti, J.P. Viola, A. Vieira-de-Abreu, P.T. Bozza, A. Sher, J. Scharfstein, Cutting edge: bradykinin induces IL-12 production by dendritic cells: a danger signal that drives Th1 polarization, J. Immunol. 170 (2003) 5349-5353.

[14] A. Kozik, R.B. Moore, J. Potempa, T. Imamura, M. Rapala-Kozik, J. Travis, A novel mechanism for bradykinin production at inflammatory sites. Diverse effects of a mixture of neutrophil elastase and mast cell tryptase versus tissue and plasma kallikreins on native and oxidized kininogens, J. Biol. Chem. 273 (1998) 33224-33229.

[15] S.G. Farmer, R.M. Burch, Biochemical and molecular pharmacology of kinin receptors, Annu. Rev. Pharmacol. Toxicol. 32 (1992) 511536.

[16] F. Marceau, D.R. Bachvarov, Kinin receptors, Clin. Rev. Allergy Immunol. 16 (1998) 385-401.

[17] H. Herwald, M. Collin, W. Muller-Esterl, L. Bjorck, Streptococcal cysteine proteinase releases kinins: a virulence mechanism, J. Exp. Med. 184 (1996) 665-673.

[18] H. Maeda, T. Yamamoto, Pathogenic mechanisms induced by microbial proteases in microbial infections, Biol. Chem. Hoppe Seyler 377 (1996) 217-226.

[19] T. Imamura, R.N. Pike, J. Potempa, J. Travis, Pathogenesis of periodontitis: a major arginine-specific cysteine proteinase from Porphyromonas gingivalis induces vascular permeability enhancement through activation of the kallikrein/kinin pathway, J. Clin. Invest. 94 (1994) 361-367.

[20] I. Rubinstein, J. Potempa, J. Travis, X.P. Gao, Mechanisms mediating Porphyromonas gingivalis gingipain RgpA-induced oral mucosa inflammation in vivo, Infect. Immun. 69 (2001) 1199-1201. 
[21] J. Scharfstein, V. Schmitz, V. Morandi, M.M. Capella, A.P. Lima, A. Morrot, L. Juliano, W. Muller-Esterl, Host cell invasion by Trypanosoma cruzi is potentiated by activation of bradykinin $\mathrm{B}(2)$ receptors, J. Exp. Med. 192 (2000) 1289-1300.

[22] E. Del Nery, M.A. Juliano, A.P. Lima, J. Scharfstein, L. Juliano, Kininogenase activity by the major cysteinyl proteinase (cruzipain) from Trypanosoma cruzi, J. Biol. Chem. 272 (1997) 25713-25718.

[23] A.P. Lima, P.C. Almeida, I.L. Tersariol, V. Schmitz, A.H. Schmaier, L. Juliano, I.Y. Hirata, W. Muller-Esterl, J.R. Chagas, J. Scharfstein, Heparan sulfate modulates kinin release by Trypanosoma cruzi through the activity of cruzipain, J. Biol. Chem. 277 (2002) 58755881

[24] A.G. Todorov, D. Andrade, J.B. Pesquero, C. Araujo Rde, M. Bader, J. Stewart, L. Gera, W. Muller-Esterl, V. Morandi, R.C. Goldenberg, H.C. Neto, J. Scharfstein, Trypanosoma cruzi induces edematogenic responses in mice and invades cardiomyocytes and endothelial cells in vitro by activating distinct kinin receptor (B1/B2) subtypes, FASEB J. 17 (2003) 73-75

[25] G.F. Spath, S.M. Beverley, A lipophosphoglycan-independent method for isolation of infective Leishmania metacyclic promastigotes by density gradient centrifugation, Exp. Parasitol. 99 (2001) 97-103.

[26] E. Svensjo, F.Z. Cyrino, L. Juliano, J. Scharfstein, Plasma leakage induced in postcapillary venules by the major cysteine-proteinase from Trypanosoma cruzi and its modulation by H1-blocker mepyramine, Microvasc. Res. 54 (1997) 93-97.

[27] E. Svensjo, Bradykinin and prostaglandin E1, E2 and F2alphainduced macromolecular leakage in the hamster cheek pouch, Prostaglandins Med. 1 (1978) 397-410.

[28] J.C. Engel, P.S. Doyle, I. Hsieh, J.H. McKerrow, Cysteine protease inhibitors cure an experimental Trypanosoma cruzi infection, J. Exp. Med. 188 (1998) 725-734.

[29] J. Ferreira, M.M. Campos, R. Araujo, M. Bader, J.B. Pesquero, J.B. Calixto, The use of kinin $\mathrm{B} 1$ and $\mathrm{B} 2$ receptor knockout mice and selective antagonists to characterize the nociceptive responses caused by kinins at the spinal level, Neuropharmacology 43 (2002) 11881197

[30] J. Kaufmann, M. Haasemann, S. Modrow, W. Muller-Esterl, Structural dissection of the multidomain kininogens. Fine mapping of the target epitopes of antibodies interfering with their functional properties, J. Biol. Chem. 268 (1993) 9079-9091.

[31] H. Goto, J.A. Lindoso, Immunity and immunosuppression in experimental visceral leishmaniasis, Braz. J. Med. Biol. Res. 37 (2004) 615-623.

[32] E. Svensjo, The hamster cheek pouch as a model in microcirculation research, Eur. Respir. J. (Suppl 12) (1990) 595s-600s (discussion 600s-601s).

[33] N.J. Brown, D.E. Vaughan, Angiotensin-converting enzyme inhibitors, Circulation 97 (1998) 1411-1420.

[34] C.M. Rao, P. Salotra, K. Datta, Possible role of the 34-kDa hyaluronic acid-binding protein in visceral Leishmaniasis, J. Parasitol. 85 (1999) 682-687.

[35] J. Scharfstein, Activation of bradykinin-receptors by Trypanosoma cruzi: a role for cruzipain in microvascular pathology, in: J. Kelly (Ed.), Molecular Mechanisms in the Pathogenesis of Chagas Disease, Landes Bioscience, Austin, Texas, USA, 2003.

[36] V. Mundodi, A. Somanna, P.J. Farrell, L. Gedamu, Genomic organization and functional expression of differentially regulated cysteine protease genes of Leishmania donovani complex, Gene 282 (2002) 257-265.
[37] R. Ramamoorthy, J.E. Donelson, K.E. Paetz, M. Maybodi, S.C. Roberts, M.E. Wilson, Three distinct RNAs for the surface protease gp63 are differentially expressed during development of Leishmania donovani chagasi promastigotes to an infectious form, J. Biol. Chem. 267 (1992) 1888-1895.

[38] Z. Shariat-Madar, F. Mahdi, A.H. Schmaier, Mapping binding domains of kininogens on endothelial cell cytokeratin 1, J. Biol. Chem. 274 (1999) 7137-7145.

[39] T. Renne, J. Dedio, G. David, W. Muller-Esterl, High molecular weight kininogen utilizes heparan sulfate proteoglycans for accumulation on endothelial cells, J. Biol. Chem. 275 (2000) 33688-33696.

[40] A.A. Hasan, D.B. Cines, H. Herwald, A.H. Schmaier, W. MullerEsterl, Mapping the cell binding site on high molecular weight kininogen domain 5, J. Biol. Chem. 270 (1995) 19256-19261.

[41] M.D. Laurenti, C.E. Corbett, M.N. Sotto, I.L. Sinhorini, H. Goto, The role of complement in the acute inflammatory process in the skin and in host-parasite interaction in hamsters inoculated with Leishmania (Leishmania) chagasi, Int. J. Exp. Pathol. 77 (1996) 15-24.

[42] M.V. Lonardoni, M. Russo, S. Jancar, Essential role of plateletactivating factor in control of Leishmania (Leishmania) amazonensis infection, Infect. Immun. 68 (2000) 6355-6361.

[43] E. Svensjo, W.L. Joyner, The effects of intermittent and continuous stimulation of microvessels in the cheek pouch of hamsters with histamine and bradykinin on the development of venular leaky sites, Microcirc. Endothelium Lymphatics 1 (1984) 381-396.

[44] S. Shigematsu, S. Ishida, D.C. Gute, R.J. Korthuis, Concentrationdependent effects of bradykinin on leukocyte recruitment and venular hemodynamics in rat mesentery, Am. J. Physiol. 277 (1999) H152H160.

[45] M. Erlansson, D. Bergqvist, N.H. Persson, E. Svensjo, Modification of postischemic increase of leukocyte adhesion and vascular permeability in the hamster by Iloprost, Prostaglandins 41 (1991) 157-168.

[46] S. Shigematsu, S. Ishida, D.C. Gute, R.J. Korthuis, Bradykinininduced proinflammatory signaling mechanisms, Am. J. Physiol. Heart Circ. Physiol. 283 (2002) H2676-H2686.

[47] C. Matte, M. Olivier, Leishmania-induced cellular recruitment during the early inflammatory response: modulation of proinflammatory mediators, J. Infect. Dis. 185 (2002) 673-681.

[48] P. Kropf, N. Freudenberg, C. Kalis, M. Modolell, S. Herath, C. Galanos, M. Freudenberg, I. Muller, Infection of $\mathrm{C} 57 \mathrm{BL} / 10 \mathrm{ScCr}$ and C57BL/10ScNCr mice with Leishmania major reveals a role for Toll-like receptor 4 in the control of parasite replication, J. Leukoc. Biol. 76 (2004) 48-57.

[49] J. Bjork, T.E. Hugli, G. Smedegard, Microvascular effects of anaphylatoxins C3a and C5a, J. Immunol. 134 (1985) 1115-1119.

[50] J.C. Mottram, D.R. Brooks, G.H. Coombs, Roles of cysteine proteinases of trypanosomes and Leishmania in host-parasite interactions, Curr. Opin. Microbiol. 1 (1998) 455-460.

[51] J. Friedland, C. Setton, E. Silverstein, Induction of angiotensin converting enzyme in human monocytes in culture, Biochem. Biophys. Res. Commun. 83 (1978) 843-849.

[52] J.M. Blackwell, R.A. Ezekowitz, M.B. Roberts, J.Y. Channon, R.B. Sim, S. Gordon, Macrophage complement and lectin-like receptors bind Leishmania in the absence of serum, J. Exp. Med. 162 (1985) 324-331.

[53] K.R. Gantt, S. Schultz-Cherry, N. Rodriguez, S.M. Jeronimo, E.T. Nascimento, T.L. Goldman, T.J. Recker, M.A. Miller, M.E. Wilson, Activation of TGF-beta by Leishmania chagasi: importance for parasite survival in macrophages, J. Immunol. 170 (2003) 2613-2620. 